\title{
ARES IV: Probing the Atmospheres of the Two Warm Small Planets HD 106315c and HD 3167c with the HST/WFC3 Camera*
}

\author{
Gloria Guilluy ${ }^{1,2}\left(\mathbb{D}\right.$, Amélie Gressier ${ }^{3,4,5}$ (D) , Sam Wright $^{6}$, Alexandre Santerne ${ }^{7}\left(\mathbb{D}\right.$, Adam Yassin Jaziri ${ }^{8}$, Billy Edwards ${ }^{6}$ (i),

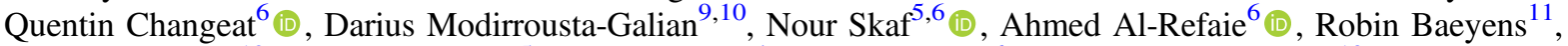

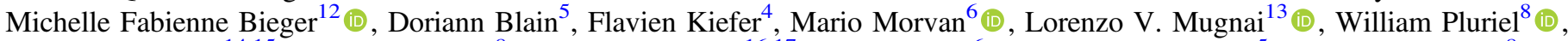

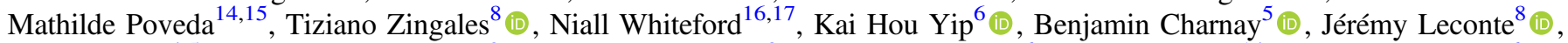

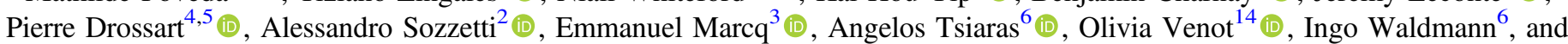 \\ Jean-Philippe Beaulieu ${ }^{4,18}$ (1) \\ ${ }^{1}$ Dipartimento di Fisica, Università degli Studi di Torino, via Pietro Giuria 1, I-10125 Torino, Italy; gloria.guilluy@inaf.it \\ ${ }^{2}$ INAF Osservatorio Astrofisico di Torino, Via Osservatorio 20, I-10025 Pino Torinese, Italy \\ ${ }^{3}$ LATMOS, CNRS, Sorbonne Université /UVSQ, 11 boulevard dAlembert, F-78280 Guyancourt, France \\ ${ }_{5}^{4}$ Sorbonne Universités, UPMC Université Paris 6 et CNRS, UMR 7095, Institut d'Astrophysique de Paris, 98 bis bd Arago, F-75014 Paris, France \\ ${ }^{5}$ LESIA, Observatoire de Paris, Université PSL, CNRS, Sorbonne Université, Université de Paris, 5 place Jules Janssen, F-92195 Meudon, France \\ ${ }^{6}$ Department of Physics and Astronomy, University College London, London, UK \\ 7 Aix Marseille Univ, CNRS, CNES, LAM, Marseille, France \\ ${ }^{8}$ Laboratoire d'astrophysique de Bordeaux, Univ. Bordeaux, CNRS, B18N, allée Geoffroy Saint-Hilaire, 33615 Pessac, France \\ ${ }^{9}$ INAF-Osservatorio Astronomico di Palermo, Piazza del Parlamento 1, I-90134 Palermo, Italy \\ ${ }^{10}$ University of Palermo, Department of Physics and Chemistry, Via Archirafi 36, Palermo, Italy \\ ${ }^{11}$ Instituut voor Sterrenkunde, KU Leuven, Celestijnenlaan 200D bus 2401, B-3001 Leuven, Belgium \\ ${ }^{12}$ College of Engineering, Mathematics and Physical Sciences, Physics Building, University of Exeter North Park Road, Exeter, UK \\ ${ }^{13}$ La Sapienza Universitá di Roma, Department of Physics, Piazzale Aldo Moro 2, I-00185 Roma, Italy \\ ${ }^{14}$ Laboratoire Interuniversitaire des Systèmes Atmosphériques (LISA), UMR CNRS 7583, Université Paris-Est-Créteil, Université de Paris, Institut Pierre Simon \\ Laplace, Créteil, France \\ ${ }^{15}$ Maison de la Simulation, CEA, CNRS, Univ. Paris-Sud, UVSQ, Université Paris-Saclay, F-91191 Gif-sur-Yvette, France \\ ${ }^{16}$ Institute for Astronomy, University of Edinburgh, Blackford Hill, Edinburgh, EH9 3HJ, UK \\ ${ }^{17}$ Centre for Exoplanet Science, University of Edinburgh, Edinburgh, EH9 3FD, UK \\ ${ }^{18}$ School of Physical Sciences, University of Tasmania, Private Bag 37 Hobart, Tasmania 7001, Australia \\ Received 2020 July 31; revised 2020 October 15; accepted 2020 October 20; published 2020 December 9
}

\begin{abstract}
We present an atmospheric characterization study of two medium-sized planets bracketing the radius of Neptune: HD 106315c $\left(R_{\mathrm{P}}=4.98 \pm 0.23 R_{\oplus}\right)$ and HD 3167c $\left(R_{\mathrm{P}}=2.740_{-0.100}^{+0.106} R_{\oplus}\right)$. We analyze spatially scanned spectroscopic observations obtained with the G141 grism $(1.125-1.650 \mu \mathrm{m})$ of the Wide Field Camera 3 (WFC3) on board the Hubble Space Telescope. We use the publicly available Iraclis pipeline and TauREx3 atmospheric retrieval code and detect water vapor in the atmosphere of both planets, with an abundance of $\log _{10}\left[\mathrm{H}_{2} \mathrm{O}\right]=-2.1_{-1.3}^{+0.7} \quad(\sim 5.68 \sigma)$ and $\log _{10}\left[\mathrm{H}_{2} \mathrm{O}\right]=-4.1_{-0.9}^{+0.9} \quad(\sim 3.17 \sigma)$ for HD 106315c and HD 3167c, respectively. The transmission spectrum of $\mathrm{HD} 106315 \mathrm{c}$ also shows possible evidence of ammonia absorption $\left(\log _{10}\left[\mathrm{NH}_{3}\right]=-4.3_{-2.0}^{+0.7}, \sim 1.97 \sigma\right.$, even if it is not significant), while carbon dioxide absorption features may be present in the atmosphere of $\mathrm{HD} 3167 \mathrm{c}$ in the $\sim 1.1-1.6 \mu \mathrm{m}$ wavelength range $\left(\log _{10}\left[\mathrm{CO}_{2}\right]=-2.4_{-1.0}^{+0.7}, \sim 3.28 \sigma\right)$. However, the $\mathrm{CO}_{2}$ detection appears significant, and it must be considered carefully and put into perspective. Indeed, $\mathrm{CO}_{2}$ presence is not explained by $1 \mathrm{D}$ equilibrium chemistry models, and it could be due to possible systematics. The additional contributions of clouds, $\mathrm{CO}$, and $\mathrm{CH}_{4}$ are discussed. $\mathrm{HD} 106315 \mathrm{c}$ and $\mathrm{HD} 3167 \mathrm{c}$ will be interesting targets for upcoming telescopes such as the James Webb Space Telescope and the Atmospheric Remote-sensing Infrared Exoplanet Large-survey.
\end{abstract}

Unified Astronomy Thesaurus concepts: Astronomy data analysis (1858); Exoplanets (498); Exoplanet atmospheres (487); Hubble Space Telescope (761)

\section{Introduction}

High-precision photometry with NASA's Kepler space mission revealed the existence of a large population of transiting planets with radii between those of Earth and Neptune and with periods shorter than 100 days (e.g., Borucki et al. 2011; Howard et al. 2012; Batalha et al. 2013; Dressing \& Charbonneau 2013; Fressin et al. 2013; Petigura et al. 2013). Thanks to more precise measurements of the stellar radii of the Kepler field, first via spectroscopy (Petigura et al. 2017; Fulton et al. 2017) and then via Gaia Data Release 2 data (Fulton \&

\footnotetext{
* ARES: ARIEL Retrieval of Exoplanets School.
}

Petigura 2018), it was then discovered that the radius distribution of small planets is bimodal with a paucity of planets with radii in the range of $1.5-2 R_{\oplus}$. The right peak of this bimodal distribution $\left(2-5 R_{\oplus}\right)$ is made up of sub-Neptune (2-4 $\left.R_{\oplus}\right)$ and Neptune-type planets $\left(R \geqslant 4 R_{\oplus}\right)$. For this population of planets, a broad range of scenarios are possible, including water worlds, rocky super-Earths, and planets with H- and He-dominated atmospheres (e.g., Léger et al. 2004; Valencia et al. 2006; Rogers \& Seager 2010a, 2010b; Rogers et al. 2011; Rogers 2015; Zeng et al. 2019). Atmospheric measurements are needed to understand their composition. To date, very few atmospheric studies concerning this class of planets have been conducted (see Table 1), but a larger number 
Table 1

Planets with Size between 2 and $5 R_{\oplus}$ with Published Atmospheric Characterization Studies

\begin{tabular}{lcl}
\hline \hline Planet & Chemical Species & Reference \\
\hline GJ 3470b & $\mathrm{H}_{2} \mathrm{O}$ & Fisher \& Heng (2018); Benneke et al. (2019a) \\
& $\mathrm{H}$ & Bourrier et al. (2018) \\
\hline GJ 436 b & Flat spectrum (clouds or hazes) & Knutson et al. (2014a) \\
& $\mathrm{H}$ & Bourrier et al. (2016) \\
\hline GJ 1214b & Flat spectrum (clouds or hazes) & Kreidberg et al. (2014a) \\
\hline HD 97658b & Flat spectrum (clouds or hazes) & Knutson et al. (2014b) \\
\hline HAT-P-11 b & $\mathrm{He}$ & Allart et al. (2018); Mansfield et al. (2018) \\
& $\mathrm{H}_{2} \mathrm{O}$ & Fraine et al. (2014); Fisher \& Heng (2018); Chachan et al. (2019) \\
Chachan et al. (2019)
\end{tabular}

of observations will be necessary to put constraints on the planetary formation and migration theories and link the larger gas giants to the smaller terrestrial planets.

An element of comparison, which allows us to better understand the atmospheric physics of sub-Neptune and Neptune-type exoplanets, can be found in our solar system, more precisely in Uranus and Neptune. These ice giants can be used as (cold) templates for listing the physical phenomena present in this class of planets, and a good understanding of them would give access to more accurate extrapolations for different temperatures of the planets. One element to emphasize are the large differences in atmospheric composition between Uranus and Neptune, reviewed in Moses et al. (2020). The observability of chemical compounds is defined by equilibrium chemistry in the hot interior, modified in the upper atmosphere by transport-induced quenching and photochemistry. The dynamic activity of the planet (modelized by an eddy diffusion coefficient for simplified mixing calculations) can therefore have a direct effect on the observable composition. Such effects could have to be considered for this class of planets, especially for warm sub-Neptune and Neptune-type planets.

In this paper we analyze the transmission spectra of the Neptune-type HD 106315c and of the sub-Neptune HD 3167c, using publicly available observations from the Hubble Space Telescope (HST) Wide Field Camera 3 (WFC3) operating in its spatial scanning mode.

The first small warm planet we studied in this paper is HD $106315 \mathrm{c}$. With a mass of $14.6 \pm 4.7 M_{\oplus}$, a radius of $4.98 \pm 0.23 R_{\oplus}$, and a density of $0.65 \pm 0.23 \mathrm{~g} \mathrm{~cm}^{-3}$, it orbits its F5V host star with a period of $21.05731 \pm 0.00046$ days (this work, Table 2). Its equilibrium temperature, computed by assuming an albedo of 0.2 (Crossfield \& Kreidberg 2017), is $835 \pm 20 \mathrm{~K}$. The planet has an inner-smaller companion HD $106315 \mathrm{~b}\left(R_{\mathrm{P}}=2.18 \pm 0.33 R_{\oplus}\right.$; this work $)$. The discovery of this multiplanetary system was simultaneously announced by Crossfield et al. (2017) and Rodriguez et al. (2017) using data from the $\mathrm{K} 2$ mission. Due to the paucity of radial velocity measurements, both teams were not able to derive a precise measurement of the planetary mass, and only the High Accuracy Radial velocity Planet Searcher (HARPS) radial velocity observations by Barros et al. (2017) allowed a mass estimation. More recently, Zhou et al. (2018) reported also an obliquity measurement $\left(\lambda=-10^{\circ} .9_{-3.8}^{+3.6}\right)$ for HD $106315 \mathrm{c}$ from Doppler tomographic observations gathered with the Magellan Inamori Kyocera Echelle (MIKE), HARPS, and the Tillinghast
Table 2

Stellar and Planetary Parameters Used in Our Analysis

\begin{tabular}{lcc}
\hline \hline Parameters & HD 106315c & HD 3167c \\
\hline \multicolumn{3}{c}{ Stellar Parameters } \\
\hline Stellar type & $\mathrm{F}^{\mathrm{a}}$ & $\mathrm{K} 0 \mathrm{~V}$ \\
{$[\mathrm{Fe} / \mathrm{H}]_{\star}$} & $-0.276 \pm 0.083$ & $0.03 \pm 0.03$ \\
$T_{\text {eff }}(\mathrm{K})$ & $6256 \pm 51$ & $5286 \pm 40$ \\
$\log _{10} g_{\star}(\mathrm{cgs})$ & $4.235 \pm 0.030$ & $4.53 \pm 0.03$ \\
$R_{\star}\left(R_{\odot}\right)$ & $1.31 \pm 0.04$ & $0.835 \pm 0.026$ \\
$M_{\star}\left(M_{\odot}\right)$ & $1.079 \pm 0.037$ & $0.877 \pm 0.024$
\end{tabular}

\begin{tabular}{lcc}
\hline \multicolumn{3}{c}{ Planetary and Transit Parameters } \\
\hline$M_{\mathrm{P}}\left(M_{\oplus}\right)$ & $14.6 \pm 4.7$ & $8.33_{-1.85}^{+1.79}$ \\
$R_{\mathrm{P}} / R_{\star}(\%)$ & $3.481 \pm 0.099$ & $3.006_{-0.055}^{+0.065}$ \\
$R_{\mathrm{P}}\left(R_{\oplus}\right)$ & $4.98 \pm 0.23$ & $2.740_{-0.100}^{+0.100}$ \\
$P$ (days) & $21.05731 \pm 0.00046$ & $29.84622_{-0.00091}^{+0.00098}$ \\
$i$ (deg) & $88.17 \pm 0.11$ & $89.6 \pm 0.2$ \\
$a / R_{\star}$ & $25.10 \pm 0.79$ & $46.5 \pm 1.5$ \\
$T_{0}\left(\mathrm{BJD}_{\mathrm{TDB}}\right)$ & $2457569.0211 \pm 0.0053$ & $2457394.97831 \pm 0.00085$ \\
$\mathrm{e}$ & $0.052 \pm 0.052$ & $0.05_{-0.04}^{+0.07}$ \\
$\omega$ & $157 \pm 140$ & $178_{-136}^{+134}$ \\
\hline
\end{tabular}

Reference $\quad$ This work, Section $2 \quad$ Gandolfi et al. (2017)

Note.

${ }^{\mathrm{a}}$ Houk \& Swift (1999).

Reflector Echelle Spectrograph (TRES). Given the brightness of the host star $(V=8.951 \pm 0.018$ mag; Crossfield et al. 2017), the atmospheric scale height $(H \sim 518 \pm 174 \mathrm{~km}$, calculated by assuming a primary mean molecular weight of $2.3 \mathrm{amu}$ ), and the contribution to the transit depth of 1 scale height $(40 \pm 14 \mathrm{ppm}$, calculated by using the relationship that the change in transit depth due to a molecular feature scales as $2 H R_{\mathrm{p}} / R_{\star}^{2}$; Brown et al. 2001), HD $106315 \mathrm{c}$ represents a golden target on which to perform transmission spectroscopy and thus to provide constraints on not only the planetary interior but also the formation and evolution history.

The other small-size planet we analyzed in this work is HD 3167c. It was discovered orbiting its host star, together with an inner planet $\mathrm{HD} 3167 \mathrm{~b}\left(R_{\mathrm{P}}=1.574 \pm 0.054 R_{\oplus}\right)$, by Vanderburg et al. (2016). Gandolfi et al. (2017) and Christiansen et al. (2017) then revised the system parameters and determined radii and masses for the two exoplanets. 
HD 3167c has a mass of $M_{\mathrm{P}}=8.33_{-1.85}^{+1.79} M_{\oplus}$, a radius of $R_{P}=2.740_{-0.100}^{+0.106} R_{\oplus}$ (Gandolfi et al. 2017), and a temperature of $T_{\text {eq }}=518 \pm 12 \mathrm{~K}$ (assuming an albedo of 0.2). It orbits its K0V host star with a period of $29.84622_{-0.00091}^{+0.00098}$ days. Given a mean density of $\rho=2.21_{-0.53}^{+0.56} \mathrm{~g} \mathrm{~cm}^{-3}$, Gandolfi et al. (2017) quoted that HD 3167c should have had a solid core surrounded by a thick atmosphere. The brightness of the host star ( $V=8.94 \pm 0.02 \mathrm{mag} ;$ Vanderburg et al. 2016), combined with the atmospheric scale height $(171 \pm 40 \mathrm{~km}$, calculated by assuming a primary mean molecular weight of $2.3 \mathrm{amu}$ ) and with the contribution to the transit depth of one scale height (18 \pm 4 ppm; this work), makes the planet a suitable target for atmospheric characterization.

We used the publicy available Python package Iraclis (Tsiaras et al. 2018) to analyze the raw HST/WFC3 images of the two warm small planets. In Section 2 we present the different steps we performed to obtain our 1D transmission spectra from the raw images. We then explain (Section 3) the modeling of the extracted spectra carried out by using the publicly available spectral retrieval algorithm TauREx3 (Waldmann et al. 2015b, 2015a; Al-Refaie et al. 2019). In Section 4, we discuss our findings and we highlight some possible limitations of our data analysis and due to WFC3's narrow spectral coverage. We also draw some interpretations of the interior compositions of the two exoplanets, and we put our results in comparison with other low spectral resolution studies (e.g., those arising from ARES; Edwards et al. 2020; Pluriel et al. 2020a; Skaf et al. 2020). We then simulate possible future studies with the upcoming space-borne instruments, such as the James Webb Space Telescope (JWST) and the Atmospheric Remote-sensing Infrared Exoplanet Large-survey (ARIEL). Finally, we conclude (Section 5) by highlighting the importance of future atmospheric characterization both from the ground and from space.

\section{Data Analysis}

From the comparison of the above-mentioned papers (Crossfield et al. 2017; Barros et al. 2017; Rodriguez et al. 2017; Zhou et al. 2018) a discrepancy emerges in the lightcurve parameters of HD $106315 \mathrm{c}$, and in particular in the value of the planetary radius $\left(R_{\mathrm{P}}\right)$. On one hand, the photometric studies by Crossfield et al. (2017), Rodriguez et al. (2017), and Barros et al. (2017) seem to converge toward a lower planetary radius $\left(\sim 4 R_{\oplus}\right)$, but with big error bars (this is probably a consequence of having a light curve with a high impact parameter). More precisely, Crossfield et al. (2017) measured a planetary radius of $3.95_{-0.39}^{+0.42} R_{\oplus}$, Rodriguez et al. (2017) one of $4.40_{-0.27}^{+0.25} R_{\oplus}$, and Barros et al. (2017) one of $4.35 \pm 0.23 R_{\oplus}$. On the other hand, the independent spectroscopic analysis by Zhou et al. (2018) resulted in a higher $R_{\mathrm{P}}$ value with smaller uncertainties (i.e., $R_{\mathrm{P}}=4.786 \pm 0.090 R_{\oplus}$ ). To overcome these inconsistencies, before looking at the HD $106315 \mathrm{c}$ 's HST/WFC3 data, we decided to perform a combined analysis, using both spectroscopic and photometric observations. More precisely, we included in our analysis ESO/HARPS radial velocities (Barros et al. 2017), space-based K2 data, and three ground-based transits, namely, one observation gathered with the Las Cumbres Observatory (LCO) telescopes (Barros et al. 2017) and two with the EULER telescope (Lendl et al. 2017). We modeled these data by employing the Markov Chain Monte Carlo Bayesian Planet Analysis and Small Transit Investigation Software (PASTIS) code (Díaz et al. 2014) as
Table 3

Proposal Information for the Data Used in Our Analysis

\begin{tabular}{lcccc}
\hline \hline Planet & Proposal ID & Proposal PI & $\begin{array}{c}\text { Transits } \\
\text { Used }\end{array}$ & $\begin{array}{c}\text { HST } \\
\text { Orbit Used }\end{array}$ \\
\hline HD 106315c & 15333 & Crossfield I. & 4 & 20 \\
HD 3167c & 15333 & Crossfield I. & 5 & 28 \\
\hline
\end{tabular}

done in Barros et al. (2017). The improved system's parameters are listed in Table 2. In particular, if we compare our results to the previous papers, trying to break the above-mentioned inconsistency on the $R_{\mathrm{P}}$ value, we note that our planetary radius is in agreement with that found by the spectroscopic analysis of Zhou et al. (2018).

Our analysis is based on four and five transit observations of HD 106315c and HD 3167c, respectively (Table 3). Both were obtained with the G141 infrared grism $(1.125-1.650 \mu \mathrm{m})$ of the HST/WFC3. The observations were part of the HST proposal GO 15333 (PI: Ian Crossfield) and were downloaded from the public Mikulski Archive for Space Telescopes (MAST) archive. An independent analysis of the same data set for HD 106315c and HD 3167c, with different pipelines, is presented by Kreidberg et al. (2020) and Mikal-Evans (2020), respectively. We analyzed and extracted white and spectral light curves from the raw HST/WFC3 images using Iraclis (Tsiaras et al. 2018). This tool includes multiple different steps:

1. Data reduction and calibration (Section 2.1)

2. Light-curve extraction (Section 2.2)

3. Limb-darkening coefficient calculation (Section 2.3)

4. White-light-curve fitting (Section 2.4)

5. Spectral light-curve fitting (Section 2.5)

Each transit was observed over six and seven HST orbits for HD 106315c and HD 3167c, respectively. We used both forward (increasing row number) and reverse (decreasing row number) scanning.

\subsection{Data Reduction and Calibration}

The first step of the Iraclis pipeline is the reduction and calibration of the HST/WFC3 raw images. This part of the analysis consists of several operations: zero-read subtraction, reference pixel correction, nonlinearity correction, dark current subtraction, gain conversion, sky background subtraction, flatfield correction, bad pixel/cosmic-ray correction, and wavelength calibration (Tsiaras et al. 2016c, 2016a, 2018).

\subsection{Light-curve Extraction}

After the reduction and calibration of the raw images, we extracted the wavelength-dependent light curves. In performing this operation, the geometric distortions caused by the tilted detector of the WFC3/IR channel are taken into account, as explained in Tsiaras et al. (2016c).

Two kinds of light curve were extracted:

1. A white light curve: calculated from a broad wavelength band $(1.088-1.68 \mu \mathrm{m})$ covering the whole wavelength range of WFC3/G141.

2. A set of spectral light curves: extracted using a narrow band with a resolving power at $1.4 \mu \mathrm{m}$ of 70 . The bins were selected such that the signal-to-noise ratio is 
Table 4

White-light-curve Fitting Results for HD 106315c and HD 106315c

\begin{tabular}{|c|c|c|c|c|c|c|c|c|c|}
\hline \multirow{2}{*}{ Planet } & \multirow{2}{*}{ Visit } & \multirow{2}{*}{$T_{0}\left(\mathrm{HJD}{ }_{-} \mathrm{UTC}\right)$} & \multirow{2}{*}{$\left(R_{\mathrm{P}} / R_{\star}\right)^{2}(\%)$} & \multicolumn{4}{|c|}{ Limb-darkening Coefficient } & \multirow{2}{*}{$n_{W}^{\text {for }}$} & \multirow{2}{*}{$n_{W}^{\mathrm{rev}}$} \\
\hline & & & & $\overline{a_{1}}$ & $a_{2}$ & $a_{3}$ & $a_{4}$ & & \\
\hline \multirow[t]{4}{*}{ HD $106315 \mathrm{c}$} & 1 & $2458453.3973_{-0.0002}^{+0.0003}$ & $0.113_{-0.002}^{+0.002}$ & \multirow[t]{4}{*}{0.8} & \multirow[t]{4}{*}{-0.8} & \multirow[t]{4}{*}{0.9} & \multirow[t]{4}{*}{-0.4} & $1341046587_{-20470}^{+30705}$ & $1340876749_{-24006}^{+27435}$ \\
\hline & 2 & $2458474.4537_{-0.0003}^{+0.0003}$ & $0.104_{-0.002}^{+0.003}$ & & & & & $1340747906_{-21939}^{+32909}$ & $1340594761_{-21530}^{+32295}$ \\
\hline & 3 & $2458516.5668_{-0.0003}^{+0.0003}$ & $0.108_{-0.003}^{+0.003}$ & & & & & $1341132987_{-35040}^{+52560}$ & $1341001153_{-35449}^{+53174}$ \\
\hline & 4 & $2458811.3661_{-0.0022}^{+0.0007}$ & $0.105_{-0.003}^{+0.003}$ & & & & & $1340514245_{-44082}^{+38572}$ & $1340404691_{-44115}^{+38600}$ \\
\hline \multirow[t]{5}{*}{ HD $3167 \mathrm{c}$} & 1 & $2458260.52574_{-0.00014}^{+0.00016}$ & $0.092_{-0.002}^{+0.002}$ & \multirow[t]{5}{*}{0.9} & \multirow[t]{5}{*}{-0.8} & \multirow[t]{5}{*}{0.9} & \multirow[t]{5}{*}{-0.4} & $1204464323_{-27844}^{+31822}$ & $1204408711_{-27166}^{+31047}$ \\
\hline & 2 & $2458320.2132_{-0.0016}^{+0.0018}$ & $0.094_{-0.002}^{+0.002}$ & & & & & $1204727344_{-32866}^{+37561}$ & $1204655124_{-32711}^{+37385}$ \\
\hline & 3 & $2458648.52966_{-0.00019}^{+0.00017}$ & $0.085_{-0.002}^{+0.003}$ & & & & & $1204169898_{-40929}^{+46776}$ & $1204128316_{-41679}^{+41679}$ \\
\hline & 4 & $2458708.220_{-0.003}^{+0.005}$ & $0.095_{-0.003}^{+0.003}$ & & & & & $1204871733_{-40165}^{+60247}$ & $1204812454_{-47121}^{+53852}$ \\
\hline & 5 & $2459036.5327_{-0.0022}^{+0.0019}$ & $0.095_{-0.001}^{+0.001}$ & & & & & $1204456150_{-19206}^{+21950}$ & $1204407529_{-18910}^{+21612}$ \\
\hline
\end{tabular}

approximately uniform across the planetary spectrum. We ended up with 25 bands, with bin widths in the range of $188.0-283.0 \mathrm{~nm}$.

\subsection{Limb-darkening Coefficients}

The stellar limb-darkening effect is modeled using the nonlinear formula with four terms from Claret (2000). The coefficients are calculated by fitting the stellar profile from an ATLAS model (Kurucz 1970; Howarth 2011) and by using the stellar parameters presented in Table 2 . Table 4 shows the limbdarkening coefficients calculated for the white light curve (between 1.125-1.650 $\mu \mathrm{m}$ ).

\subsection{White-light-curve Fitting}

The products of the previous steps are the white and spectral light curves. To continue our characterization of the two exoplanet atmospheres, we then created transmission spectra that were obtained by fitting the light curves with a transit model. However, before fitting the extracted white and spectral light curves, we had to consider the time-dependent systematics introduced by HST: one long-term "ramp" (which affects all the visits) with a linear (and, in some cases, a quadratic) trend and one short-term "ramp" (which affects every HST orbit) with an exponential trend.

In order to remove all these systematics, we fitted the white light curves using the transit Python package PyLightcurve, i.e., we used a transit model multiplied by a model for the systematics (Tsiaras et al. 2016c, 2018):

$$
n_{W}^{\text {scan }}\left[1-r_{a 1}\left(t-T_{0}\right)+r_{a 2}\left(t-T_{0}\right)^{2}\right] \cdot\left[1-r_{b_{1}} e^{-r_{b_{2}}\left(t-t_{0}\right)}\right],
$$

where $t$ is time, $T_{0}$ is the midtransit time, $t_{0}$ is the starting time of each HST orbit, $r_{a 1}$ and $r_{a 2}$ are the slopes of the linear and quadratic systematic trends, $r_{b_{1}}$ and $r_{b_{2}}$ are the exponential systematic trend's coefficients, and $n_{W}^{\text {scan }}$ is a normalization factor that changes for forward scanning $\left(n_{W}^{\text {for }}\right)$ and for reverse scanning $\left(n_{W}^{\text {rev }}\right)$. Second-order (quadratic) visit-long ramps were also fitted for HD 3167c visits because they were more affected by systematics. The parameter space was sampled via emcee (Foreman-Mackey et al. 2013). We used 300,000 emcee iterations, 200 walkers, and 100,000 burned iterations. We employed this setup for all the visits for both the planets. The only exception is represented by the fourth visit of
HD 106315 c, where we had to use 200,000 iterations to obtain a good fit to our data.

Figure 1 shows the light curves for the first transits of both exoplanets divided by the best-fit systematic model. (The same plots for the other transits are shown in Figure A1). In the fit we took $T_{0}$ and $R_{\mathrm{P}} / R_{\star}$ as free parameters, and we used fixed values for $P, \omega, i, a / R_{\star}$, and $e$ parameters, as reported in Table 2 . We made this choice because we miss ingress/egress observations in some visits. For both the planets we decided to eliminate data gathered during the first HST orbit and the first two points of each orbit because of the stronger systematics that affect them. An incorrect fitting of the behavior of the instrument at this stage would have introduced additional uncertainties in the final values of the transit parameters. Processing visits 3 and 4 for HD 3167c required additional steps; this was on account of poor initial fitting due to HD $3167 \mathrm{~b}$ also transiting the stellar disk during these observations. Strong autocorrelation in the fit residuals for visits 3 and 4 led to an investigation of the orbits for both the transiting planets in the HD 3167 system: b and c. Theoretical transit light curves were plotted for all four HD 3167 observation windows, again using PyLightcurve and taking parameters for both planets from Gandolfi et al. (2017). The theoretical light curves showed no overlap between transits for the first two visits but contamination of the third and fourth visits by concurrent transits of HD $3167 \mathrm{~b}$. In both cases this effect was limited to a single HST orbit in each affected visit. These two orbits were then disregarded, leaving six orbits for each of visits 1,2 , and 5 and five orbits apiece for visits 3 and 4. These affected orbits can be seen in Figure A2. The final fitting results and their uncertainties can be found in Table 4.

\subsection{Spectral Light-curve Fitting}

In order to correct for the systematics present in the spectral light curves, we used the divide white method introduced by Kreidberg et al. (2014a), i.e., each spectral light curve was fitted with a model that includes the white light curve and its best-fit model:

$$
n_{\lambda}^{\text {scan }}\left[1-r_{a}\left(t-T_{0}\right)\right] \frac{\mathrm{LC}_{W}}{M_{W}},
$$

where $r_{a}$ is the coefficient of a wavelength-dependent linear slope along each HST visit, $\mathrm{LC}_{W}$ is the white light curve, $M_{W}$ is the best-fitting model to the white light curve, $n_{\lambda}^{\text {scan }}$ is the normalization factor we used (it changes to $n_{\lambda}^{\text {for }}$ when the scanning direction is upward and to $n_{\lambda}^{\text {rev }}$ when it is downward). 


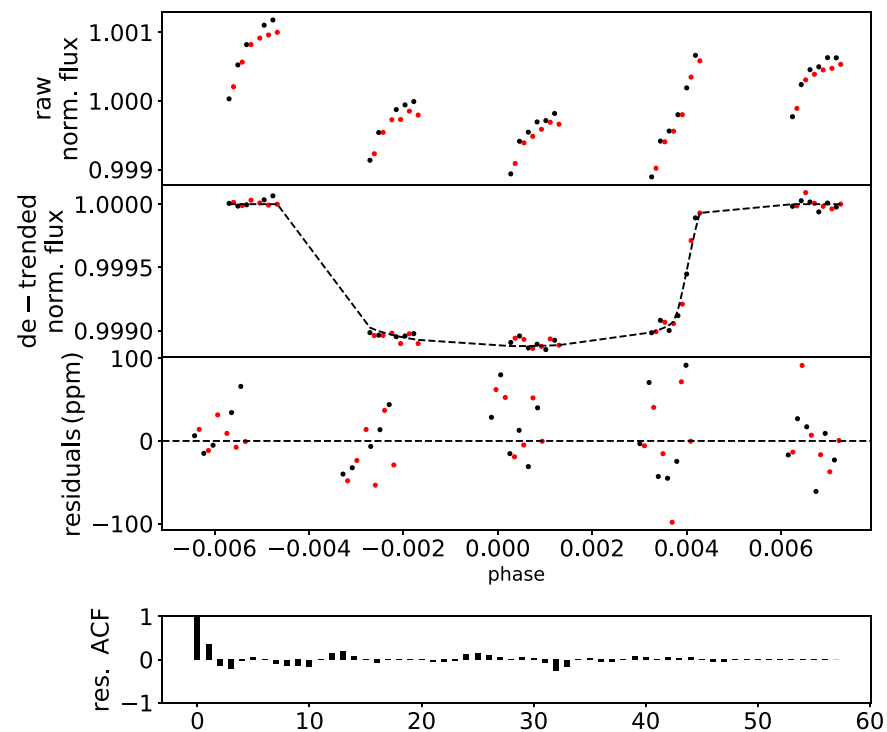

(a)

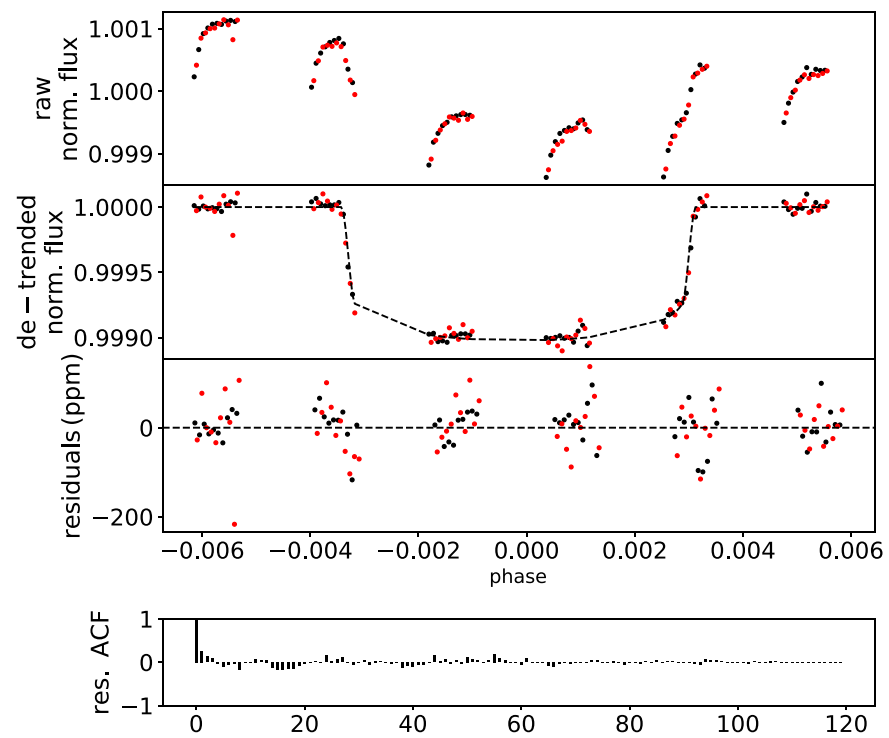

(b)

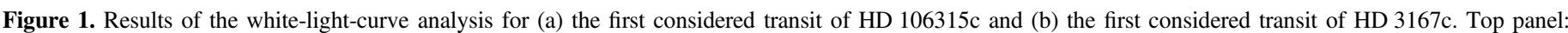

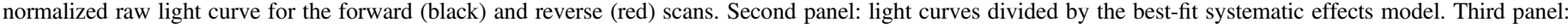
fitting residuals. Bottom panel: autocorrelation function of residuals.

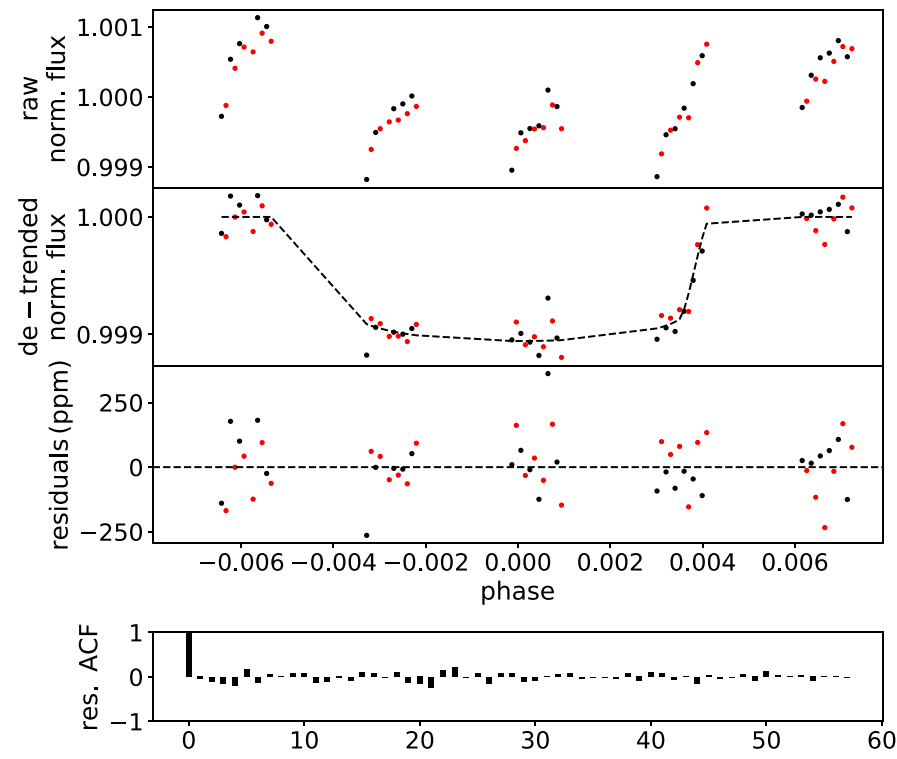

(a)

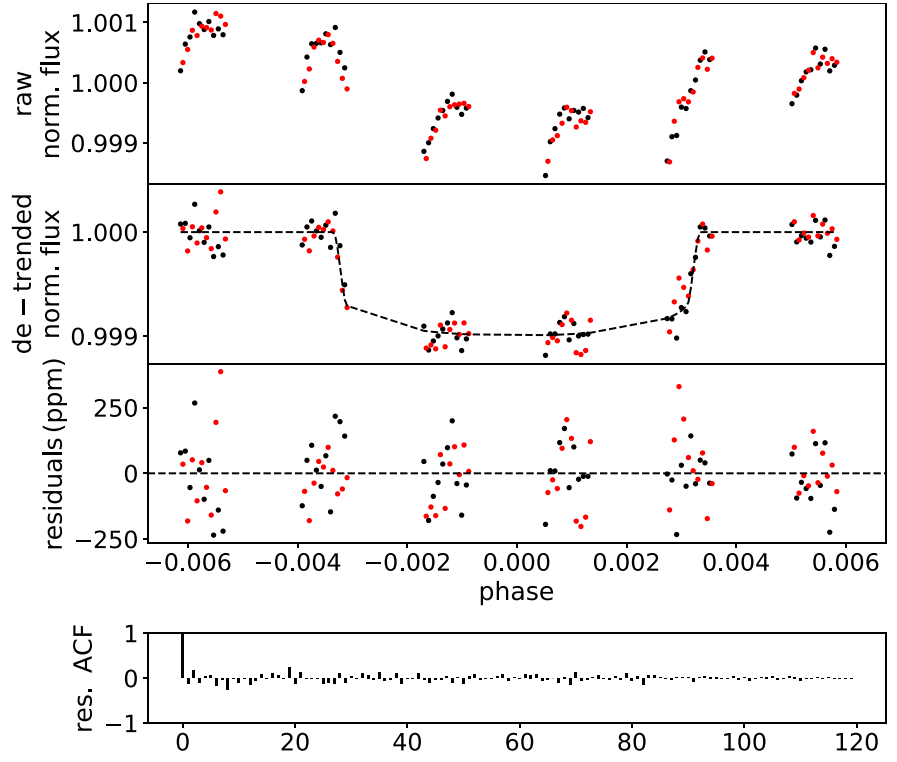

(b)

Figure 2. 10th bin $(\lambda \sim 1.3 \mu \mathrm{m})$ spectral light curve for the first transit of (a) HD 106315c and (b) HD 3167c.

As Table 4 shows, we obtained big numbers for these normalization factors; this is because the light curves are in units of electrons, and thus the large values are reasonable. In the spectral light-curve fitting, the only free parameter is $R_{\mathrm{P}} / R_{\star}$, while the other parameters are the same as we used for the white-light-curve fitting. Using the white light curve as a comparison has the advantage that the residuals from fitting one of the spectral light curves (see Figure 2) do not show trends similar to those in the white light curve (see Figure 1). All the spectral and white light curves we obtained, for the first HST visit of each planet, are plotted in Figure 3. As for the whitelight-curve fitting, the parameter space was sampled by using the emcee method. In this case we used 50,000 emcee iterations, 100 walkers, and 20,000 burned iterations.

Starting from the spectral light curves, the final spectra were extracted and combined from the spectral light curves by computing the average of the transit spectra weighted by their respective uncertainties. First, we subtracted each spectrum by 

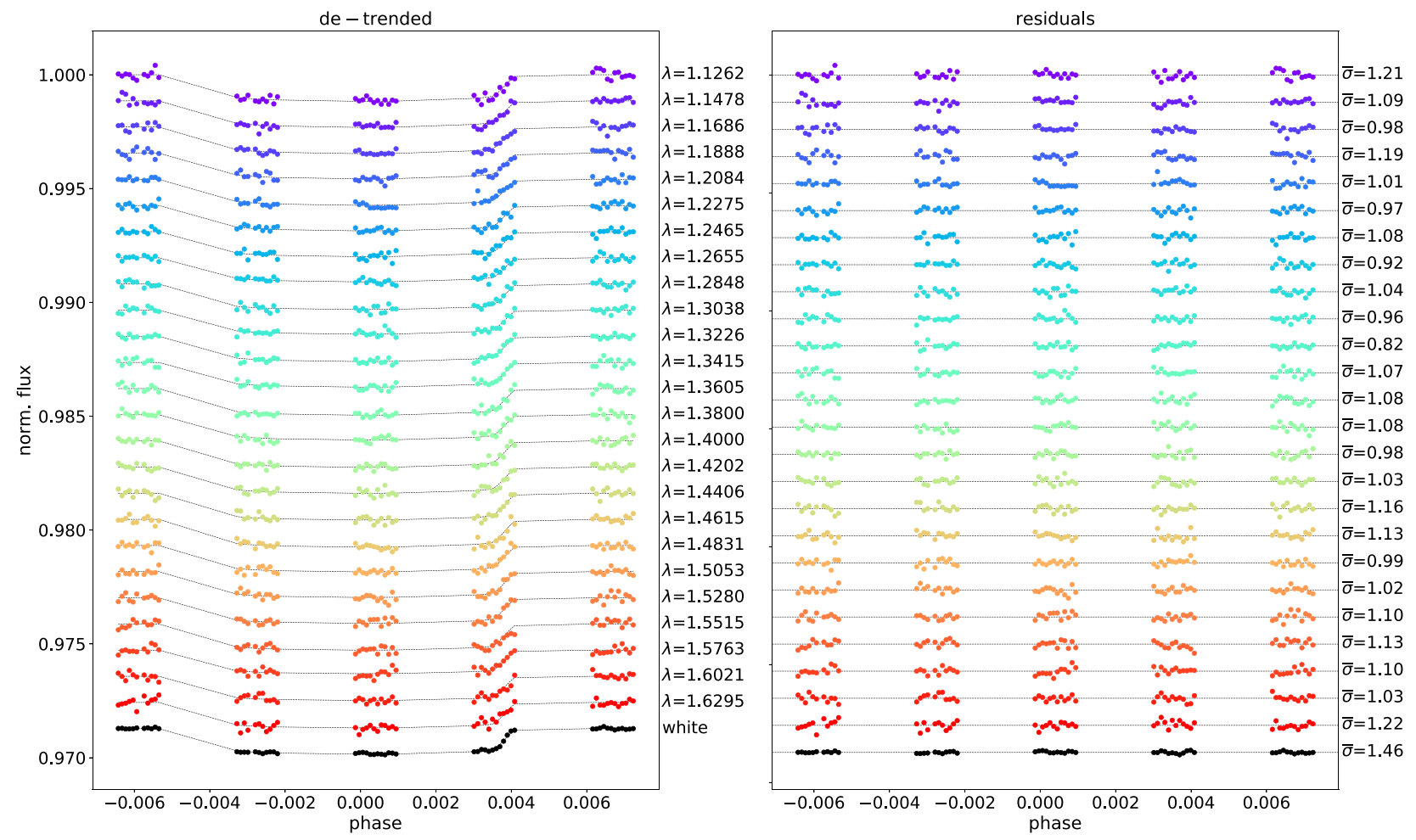

(a)
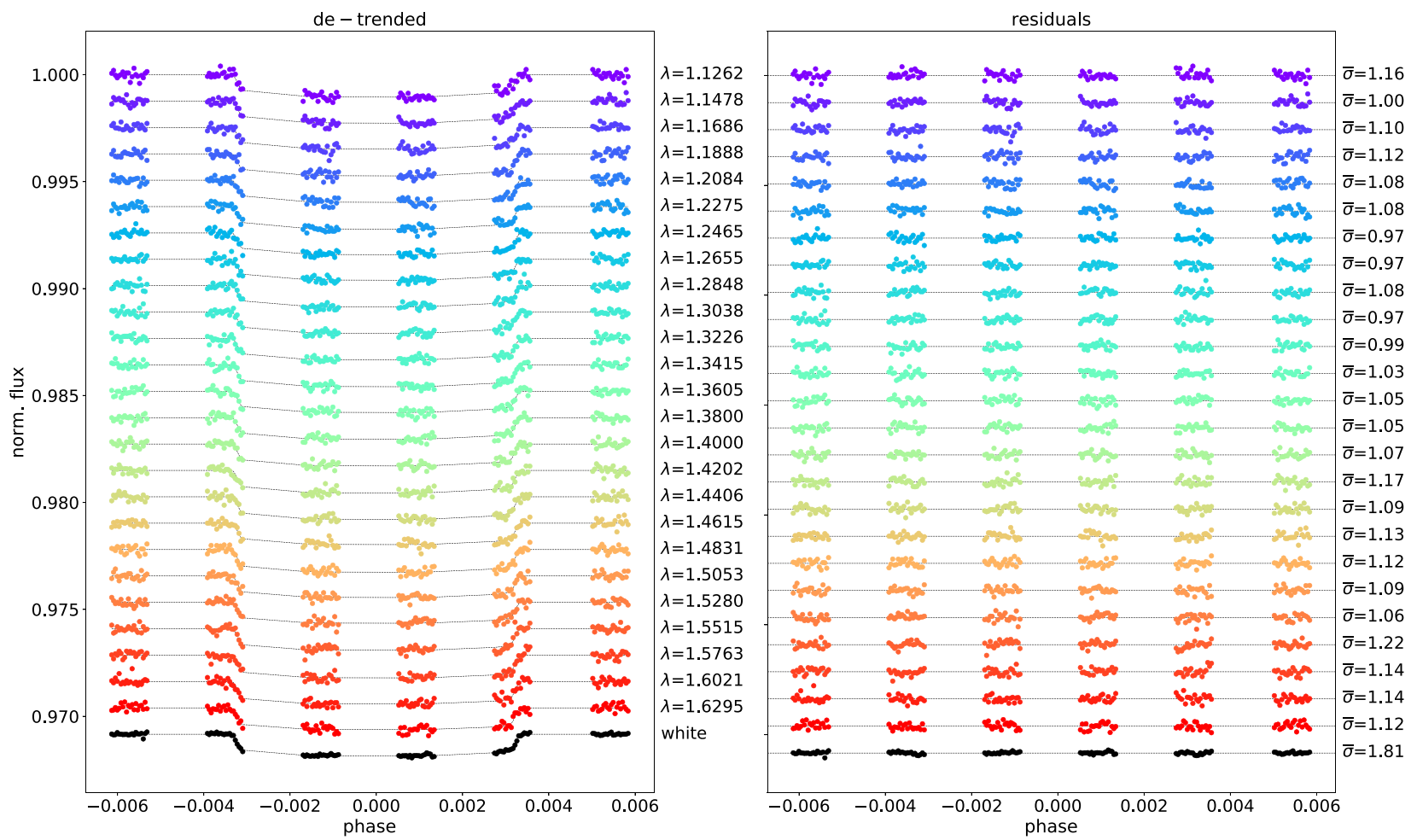

(b)

Figure 3. Analysis of the (a) HD 106315c and (b) HD 3167c white and spectral light curves (left panels), for the first transit, plotted with an offset for clarity. Left panels: overplotted white (black points) and spectral (colored points) light curves. Right panels: overplotted residuals; $\bar{\sigma}$ indicates the ratio between the standard deviation of the residuals and the photon noise. The reason that some $\bar{\sigma}$ fall below 1 is the small number of data points. Hence, the measured standard deviation is not always representative of standard deviation of the underlying distribution. These values are displayed as an indication for the goodness of fit, highlighting the differences between the different wavelengths and most importantly differences between the white light curve and spectral light curves. 
Table 5

Transit Depth $\left(R_{\mathrm{P}} / R_{\star}\right)^{2}$ for the Different Wavelength Channels, Where $R_{\mathrm{P}}$ is the Planetary Radius, $R_{\star}$ is the Stellar Radius, and $\lambda$ is the Center Value of Each Wavelength Channel

\begin{tabular}{lc}
\hline \hline & HD 106315c \\
\hline$\lambda$ & $\left(R_{\mathrm{P}} / R_{\star}\right)^{2}$ \\
$(\mu \mathrm{m})$ & $(\%)$ \\
\hline 1.1263 & $0.1064 \pm 0.0027$ \\
1.1478 & $0.1098 \pm 0.0019$ \\
1.1686 & $0.1060 \pm 0.0018$ \\
1.1888 & $0.1065 \pm 0.0019$ \\
1.2084 & $0.1068 \pm 0.0017$ \\
1.2275 & $0.1063 \pm 0.0019$ \\
1.2465 & $0.1082 \pm 0.0019$ \\
1.2655 & $0.1029 \pm 0.0018$ \\
1.2848 & $0.1078 \pm 0.0019$ \\
1.3038 & $0.1046 \pm 0.0017$ \\
1.3226 & $0.1068 \pm 0.0018$ \\
1.3415 & $0.1080 \pm 0.0019$ \\
1.3605 & $0.1130 \pm 0.0018$ \\
1.3801 & $0.1096 \pm 0.0018$ \\
1.4000 & $0.1099 \pm 0.0017$ \\
1.4202 & $0.1086 \pm 0.0017$ \\
1.4406 & $0.1130 \pm 0.0017$ \\
1.4615 & $0.1126 \pm 0.0019$ \\
1.4831 & $0.1111 \pm 0.0019$ \\
1.5053 & $0.1116 \pm 0.0017$ \\
1.5280 & $0.1074 \pm 0.0019$ \\
1.5516 & $0.1106 \pm 0.0020$ \\
1.5762 & $0.1044 \pm 0.0019$ \\
1.6021 & $0.1062 \pm 0.0019$ \\
1.6295 & $0.1018 \pm 0.0020$ \\
&
\end{tabular}

\begin{tabular}{lc}
\hline & HD 3167c \\
\hline$\lambda$ & $\left(R_{\mathrm{P}} / R_{\star}\right)^{2}$ \\
$(\mu \mathrm{m})$ & $(\%)$ \\
\hline 1.1263 & $0.0950 \pm 0.0012$ \\
1.1478 & $0.0945 \pm 0.0012$ \\
1.1686 & $0.0926 \pm 0.0012$ \\
1.1888 & $0.0924 \pm 0.0011$ \\
1.2084 & $0.0930 \pm 0.0012$ \\
1.2275 & $0.0935 \pm 0.0011$ \\
1.2465 & $0.0909 \pm 0.0011$ \\
1.2655 & $0.0915 \pm 0.0011$ \\
1.2848 & $0.0903 \pm 0.0012$ \\
1.3038 & $0.0913 \pm 0.0011$ \\
1.3226 & $0.0912 \pm 0.0011$ \\
1.3415 & $0.0920 \pm 0.0011$ \\
1.3605 & $0.0928 \pm 0.0011$ \\
1.3801 & $0.0949 \pm 0.0011$ \\
1.4000 & $0.0955 \pm 0.0011$ \\
1.4202 & $0.0961 \pm 0.0011$ \\
1.4406 & $0.0970 \pm 0.0011$ \\
1.4615 & $0.0937 \pm 0.0011$ \\
1.4831 & $0.0958 \pm 0.0012$ \\
1.5053 & $0.0925 \pm 0.0012$ \\
1.5280 & $0.0944 \pm 0.0012$ \\
1.5516 & $0.0938 \pm 0.0012$ \\
1.5762 & $0.0957 \pm 0.0012$ \\
1.6021 & $0.0937 \pm 0.0012$ \\
1.6295 & $0.0932 \pm 0.0013$ \\
\hline &
\end{tabular}

the corresponding white-light-curve depth, and then we computed the weighted average of all the transit observations. Finally, we added the weighted average of all white-light-curve

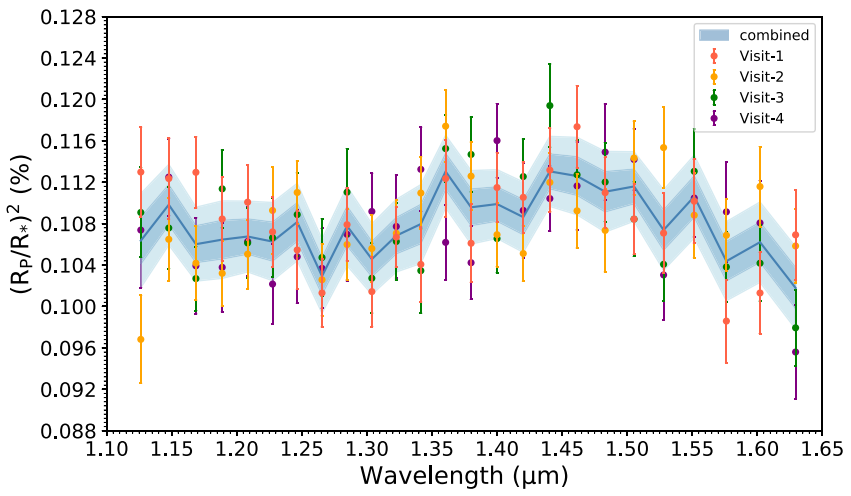

(a)

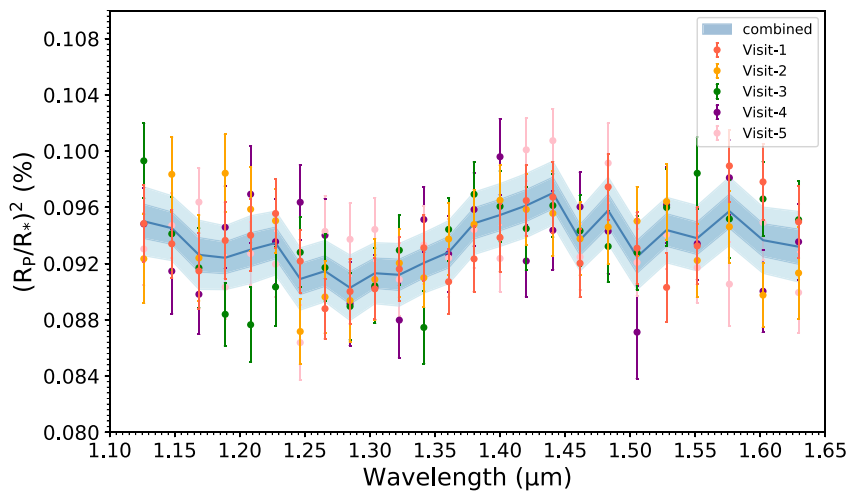

(b)

Figure 4. Spectra per visit and final weighted average with $1 \sigma$ and $2 \sigma$ uncertainty ranges for (a) HD 106315c and (b) HD 3167c.

values to the averaged spectrum. The white-light transit depths were consistent between transits, except for visit 3 for HD 3167c $(0.0291 \pm 0.0005$ compared to the weighted mean $0.03058 \pm 0.00015)$. This is probably due to remaining systematics or to stellar activity. We obtained a final spectrum with an increased signal-to-noise ratio (Table 5 and Figure 4), which we then used for atmospheric retrieval.

\section{Atmospheric Characterization}

\subsection{TauREx Setup}

Once each planetary spectrum was obtained, we fitted it using the retrieval code TauREx $3^{19}$ (Al-Refaie et al. 2019). This algorithm uses the nested sampling code Multinest (Feroz et al. 2009) to map the atmospheric forward model parameter space and find the best fit to our empirical spectra. In our retrieval analysis we used 1500 live points and an evidence tolerance of 0.5 .

The atmosphere of the two warm small planets was simulated by assuming an isothermal temperature-pressure $(T / P)$ profile with molecular abundances constant as a function of altitude. These assumptions are acceptable since, due to the short wavelength covered by HST/WFC3, we are probing a restricted range of the planetary $T / P$ profile (Tsiaras et al. 2018). We note that this may not be the case anymore with next-generation space telescopes (Rocchetto et al. 2016; Changeat et al. 2019). We calculated the equilibrium

19 https://github.com/ucl-exoplanets/TauREx3_public 
Table 6

Fit Evaluation Criteria and Maximum A Posteriori Retrieval Results

\begin{tabular}{lccc}
\hline \hline Retrieved Parameters & Bounds & HD $106315 \mathrm{c}$ & HD 3167c \\
\hline$T_{\mathrm{P}}(\mathrm{K})$ & $\pm 60 \% T_{\mathrm{eq}}$ & $630_{-115}^{+326}$ & $440_{-79}^{+119}$ \\
$R_{\mathrm{P}}\left(R_{\mathrm{J}}\right)$ & $\pm 50 \% R_{\mathrm{P}}$ & $0.395_{-0.021}^{+0.009}$ & $0.246_{-0.002}^{+0.002}$ \\
$\log _{10}\left[\mathrm{H}_{2} \mathrm{O}\right]$ & {$[-12 ;-1]$} & $-2.1_{-1.3}^{+0.7}$ & $-4.1_{-0.9}^{+0.9}$ \\
$\log _{10}\left[\mathrm{NH}_{3}\right]$ & {$[-12 ;-1]$} & $-4.3_{-2.0}^{+0.7}$ & $<-5$ \\
$\log _{10}\left[\mathrm{CO}_{2}\right]$ & {$[-12 ;-1]$} & unconstrained & $-2.4_{-1.0}^{+0.7}$ \\
$\log _{10}[\mathrm{CO}]$ & {$[-12 ;-1]$} & unconstrained & unconstrained \\
$\log _{10}\left[\mathrm{CH} \mathrm{H}_{4}\right]$ & {$[-12 ;-1]$} & $<-5$ & $<-5$ \\
$\log _{10}\left[P_{\mathrm{clouds}} / 1 \mathrm{~Pa}\right]$ & {$[-2 ; 6]$} & $3.7_{-1.3}^{+1.4}$ & $5.3_{-0.5}^{+0.5}$ \\
$\mu($ derived $)$ & & $2.38_{-0.07}^{+0.52}$ & $2.44_{-0.13}^{+0.66}$ \\
\hline $\mathrm{ADI}_{\Delta_{\mathrm{E} 2}}$ & $\ldots$ & 15.97 & 9.58 \\
$\chi^{2}$ & $\ldots$ & 6.07 & 6.65 \\
\hline$\sigma$-level & $\ldots$ & 22.35 & 24.62 \\
\hline
\end{tabular}

Note.

a The $\sigma$-level corresponds to the significance of the ADI.

temperatures of the two planets using the following formula:

$$
T_{\mathrm{eq}}=T_{\star}\left(\frac{R_{\star}}{2 a}\right)^{1 / 2}(1-A)^{1 / 4},
$$

where $R_{\star}$ is the stellar radius, $a$ is the semimajor axis, and $A$ is the geometric albedo. Assuming an albedo of 0.2 (Crossfield \& Kreidberg 2017), we obtained a temperature of $835 \pm 20 \mathrm{~K}$ and $518 \pm 12 \mathrm{~K}$ for HD $106315 \mathrm{c}$ and HD 3167c, respectively. We then used a wide range of temperature priors $\pm 60 \% T_{\text {eq }}$ (334-1336 K for HD 106315c and 207-829 K for HD 3167c) to allow different temperatures around the expected $T_{\text {eq }}$. The planetary radius is also fitted in the model ranging from $\pm 50 \%$ of the values reported in Table $2\left(0.22-0.68 R_{\mathrm{J}}\right.$ for HD $106315 \mathrm{c}$ and $0.12-0.38 R_{\mathrm{J}}$ for HD $\left.3167 \mathrm{c}\right)$.

We simulated atmospheres with pressures between $10^{-2}$ and $10^{6} \mathrm{~Pa}$, uniformly distributed in log-space across 100 planeparallel layers. We considered the trace gases $\mathrm{H}_{2} \mathrm{O}$ (Polyansky line list; Polyansky et al. 2018), $\mathrm{CH}_{4}$ (Exomol line list; Yurchenko \& Tennyson 2014), CO (line list from Li et al. 2015), $\mathrm{CO}_{2}$ (Hitemp line list; Rothman et al. 2010), and $\mathrm{NH}_{3}$ (Exomol line list; Yurchenko et al. 2011) and assumed the atmosphere to be $\mathrm{H}_{2} / \mathrm{He}$ dominated. Each trace-gas abundance was allowed to vary between $10^{-12}$ and $10^{-1}$ in volume mixing ratios (log-uniform prior). We used absorption cross sections at a resolution of 15,000 and included Rayleigh scattering and collision-induced absorption (CIA) of $\mathrm{H}_{2}-\mathrm{H}_{2}$ and $\mathrm{H}_{2}$ - $\mathrm{He}$ (Abel et al. 2011, 2012; Fletcher et al. 2018). Clouds are modeled assuming a gray opacity model, and cloud top pressure bounds are set between $10^{-2}$ and $10^{6} \mathrm{~Pa}$. All priors are listed in Table 6. Recently, Kreidberg et al. (2020) presented a transmission spectrum of HD 106315c based on HST/WFC3, $\mathrm{K} 2$, and Spitzer observations. They chose to add $\mathrm{N}_{2}$ in the retrieval analysis of HD 106315 c to compensate for invisible molecular opacities that could impact the mean molecular weight. The high equilibrium temperature of HD $106315 \mathrm{c}$ $(\sim 800 \mathrm{~K})$ suggests indeed the favored presence of $\mathrm{N}_{2}$. However, we note that no further constraints have been found regarding $\mathrm{N}_{2}$ opacity in the posterior distributions presented in Kreidberg et al. (2020). Considering this result and for consistency with HD 3167c, whose equilibrium temperature is lower $(\sim 500 \mathrm{~K})$, we decided to consider $\mathrm{NH}_{3}$ instead of $\mathrm{N}_{2}$ in the retrieval analysis for both planets. This choice is mainly motivated by the low density of HD 106315c $\left(\sim 600 \mathrm{~kg} \mathrm{~m}^{-3}\right)$, indicating, most likely, a primary light atmosphere. We therefore decided not to add $\mathrm{N}_{2}$ to the analysis in order to maintain a primary mean molecular weight ( $\mu \sim 2.3 \mathrm{amu}$ ).

To assign a significance to our detection, we used the Atmospheric Detectability Index (ADI; Tsiaras et al. 2018). It is a positively defined Bayes factor between the nominal atmospheric model and a flat-line model (a model that contains no active trace gases, Rayleigh scattering, or collision-induced absorption). We also computed two other Bayes factors in the same way as the ADI. The first one, $\Delta_{\mathrm{E} 1}$, is used to compute the significance of a molecule detection using a Bayes factor between the nominal atmospheric model and the same model without the considered molecule. The second one, $\Delta_{\mathrm{E} 2}$, compares a given model to a model containing only water, Rayleigh scattering, and collision-induced absorption as the reference Bayesian's evidence. It is used to assess the necessity of a complex model to explain the atmosphere of the observed planet. These Bayes factors were then translated into a statistical significance (Kass \& Raftery 1995) by using Table 2 of Benneke \& Seager (2013). Significances greater than 3.6 are considered "strong"; 2.7-3.6, "moderate"; 2.1-2.7, "weak"; and below 2.1, "insignificant."

\subsection{Results}

Table 6 lists our full TauREx retrieval results for the two planets, while retrieved best-fit spectra and corresponding bestfit molecular opacity contributions are shown in Figure 5. Posterior distributions are plotted in Figures 6 and 7. For each opacity source, the contribution function is the transit depth that we would obtain if the molecule was alone in the atmosphere. Therefore, the opacity sources, like $\mathrm{H}_{2} \mathrm{O}$ in HD 3167c (Figure 5(d)), are never fully dominant since there are always some residual CIA, Rayleigh, or other molecules that contribute to the model. Opacity contributions are represented for one solution, the one considered as the best one statistically speaking, i.e., with the highest log evidence. The offset opacities correspond to molecules that do not contribute to the fit and are found to be unconstrained. Besides, the gray line in Figures 5(c) and (d) represents the top cloud pressure retrieved by TauREx for the best-fit solution. The signal is theoretically blocked by this layer, and nothing can be observed at higher pressures. Opacities found below this line are unconstrained. Using the Bayesian log evidences, we computed the ADI, $\Delta_{\mathrm{E} 1}$, and $\Delta_{\mathrm{E} 2}$ as explained in Section 3.1. For both planets, retrieval results are consistent with water absorption features detectable in the spectral band covered by the G141 grism. We note a significant detection of carbonbearing species in the atmosphere of HD 3167c consistent with $\mathrm{CO}_{2}$ absorption features. This result is unexpected; indeed, considering the planetary equilibrium temperature, $\mathrm{CH}_{4}$ features are more likely to be present than $\mathrm{CO}_{2}$ (see, e.g., Figure 8 and Venot et al. 2020). Other species like $\mathrm{NH}_{3}, \mathrm{CO}$, and $\mathrm{CH}_{4}$ have either unconstrained or low abundances. They could be present in both atmospheres, but spectra do not present significant absorption features. We note, however, that $\mathrm{NH}_{3}$ abundance is better constrained in the atmosphere of HD 106315c (see Figure 6). Clouds' top pressure is retrieved at different levels, $10^{3.7} \mathrm{~Pa}$ for HD $106315 \mathrm{c}$ and $10^{5.3} \mathrm{~Pa}$ for 


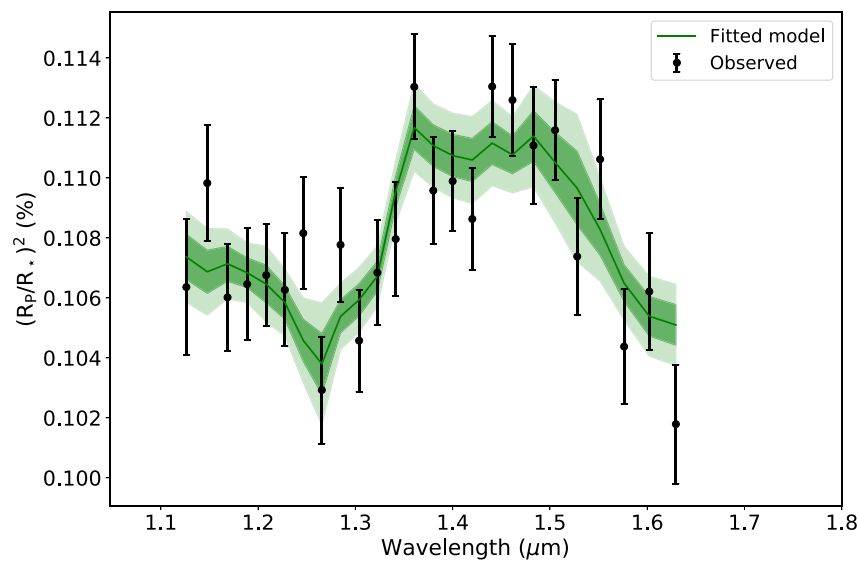

(a)

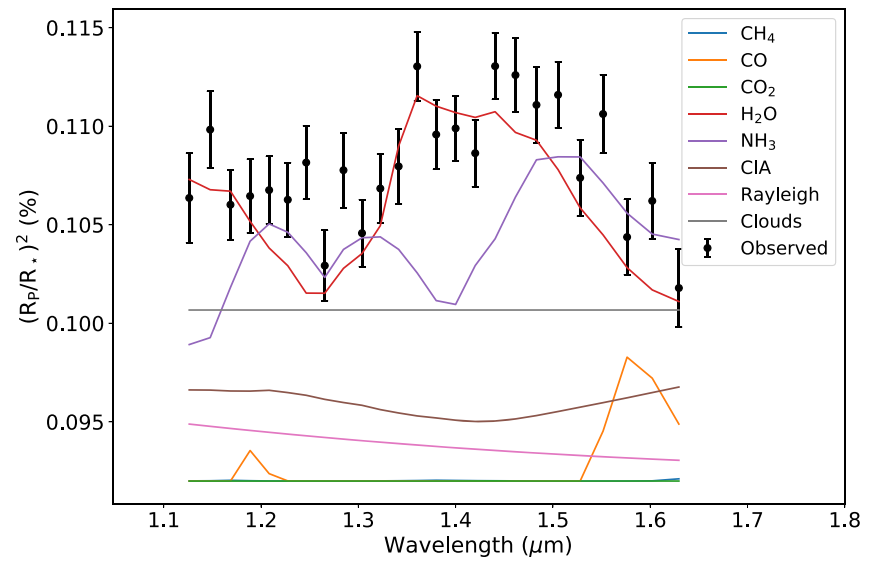

(c)

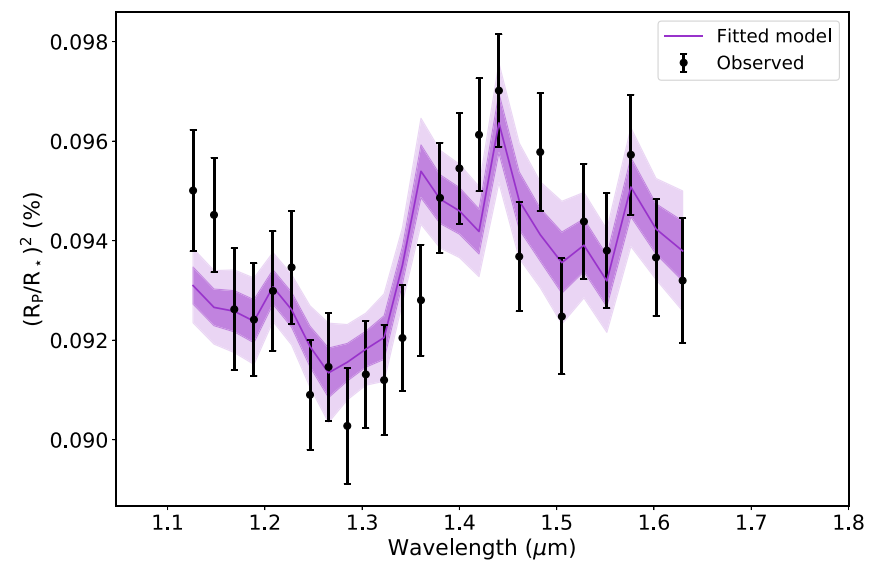

(b)

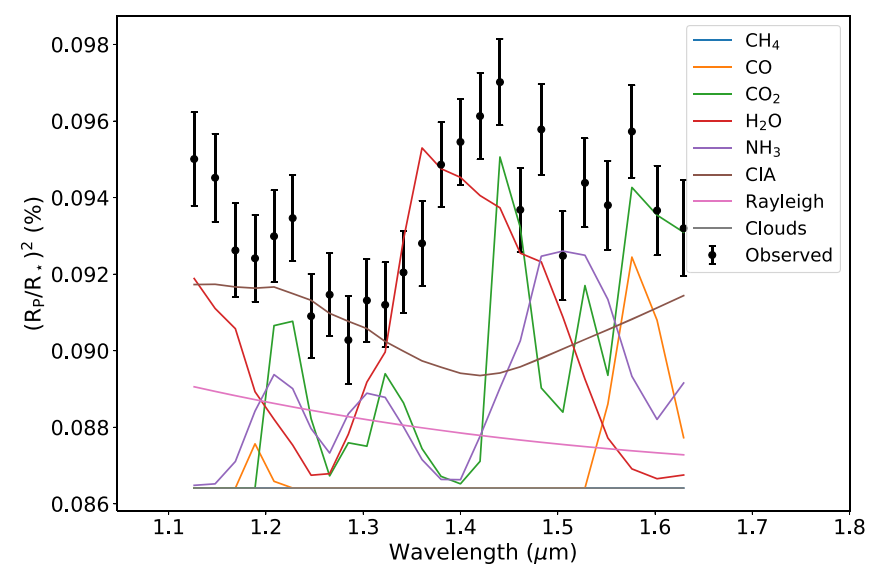

(d)

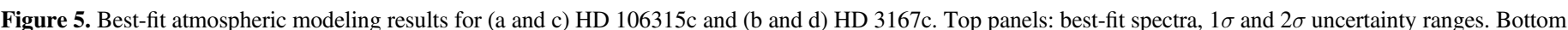

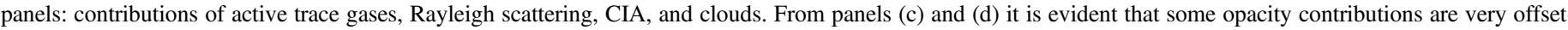
from the data. These correspond to molecules that do not contribute to the fit and are found to be unconstrained.

HD 3167c, corresponding to an upper bound (see the posterior distribution in Figures 6 and 7). The presence of molecular features in our spectra suggests a clear atmosphere for both planets. If opaque clouds are present, they are located below the region probed by WFC3/G141 observations.

\subsubsection{HD $106315 \mathrm{c}$}

According to the ADI, we retrieved a significant $(5.99 \sigma)$ atmosphere around the warm Neptune HD 106315c with a notable water detection. $\mathrm{H}_{2} \mathrm{O}$ is the only species that explains the absorption features between 1.3 and $1.5 \mu \mathrm{m}$ (Figure 5). We obtained a temperature of $630_{-115}^{+326} \mathrm{~K}$, which is lower than the equilibrium temperature, but consistent within $1 \sigma$. This could be explained by the fact that we are probing the atmosphere in the terminator area, and we modeled the atmosphere in 1D using an isothermal profile (Caldas et al. 2019; MacDonald et al. 2020; Pluriel et al. 2020b). Skaf et al. (2020), by analyzing their three hot Jupiters (WASP-127b, WASP-79b, and WASP-62b) together with the exoplanets from Tsiaras et al. (2018), highlighted the existence of a global trend between the equilibrium and the retrieved temperatures, with the retrieved temperatures showing almost always lower values. In Figure 9 we updated Figure 6 from Skaf et al. (2020) by adding the retrieved/equilibrium temperatures of the two Neptune-like planets analyzed in this work. We can see that HD 106315c follows the global trend.

The best-fit solution contains a notable amount of water, $\log _{10}\left[\mathrm{H}_{2} \mathrm{O}\right]=-2 \cdot 1_{-1.3}^{+0.7}$. Figure 6 shows that the right wing of the water's abundance Gaussian distribution is not complete. This indicates that the abundance of $\mathrm{H}_{2} \mathrm{O}$ could take even higher values $\left(\log _{10}\left[\mathrm{H}_{2} \mathrm{O}\right] \sim-1\right)$, but this is an unrealistic solution for a primary atmosphere, expected here for this Neptune-type planet. This is due to the limited coverage of HST/WFC3 G141. We note that the Bayes factor between a pure water model and the full chemical model $\Delta_{\mathrm{E} 2}$ is equal to 6.07 (see Table 7), meaning that the complexity of the full chemical model is justified with a "strong" significance $(3.91 \sigma)$.

The temperature retrieved by TauRex $(\sim 600 \mathrm{~K})$ is compatible with absorption from $\mathrm{NH}_{3}$, and this strenghtens our choice to consider $\mathrm{NH}_{3}$ as active gas instead of $\mathrm{N}_{2}$. However, $\mathrm{NH}_{3}$ contribution is debatable-the detection is driven by a few points at $1.28,1.55$, and $1.60 \mu \mathrm{m}$, hence the weak abundance of $\log _{10}\left[\mathrm{NH}_{3}\right]=-4.3_{-2.0}^{+0.7}$. We note that a high-temperature solution gives no constraint on $\mathrm{NH}_{3}$ abundance, whereas a lower temperature requires the molecule to be present (Figure 7). $\mathrm{NH}_{3}$ abundance is also correlated to the amount of $\mathrm{H}_{2} \mathrm{O}$. Moreover, we can only put constraints on the higher abundance of $\mathrm{CH}_{4}$ : it could be found below $10^{-5}$. $\mathrm{CO}$ and $\mathrm{CO}_{2}$ 


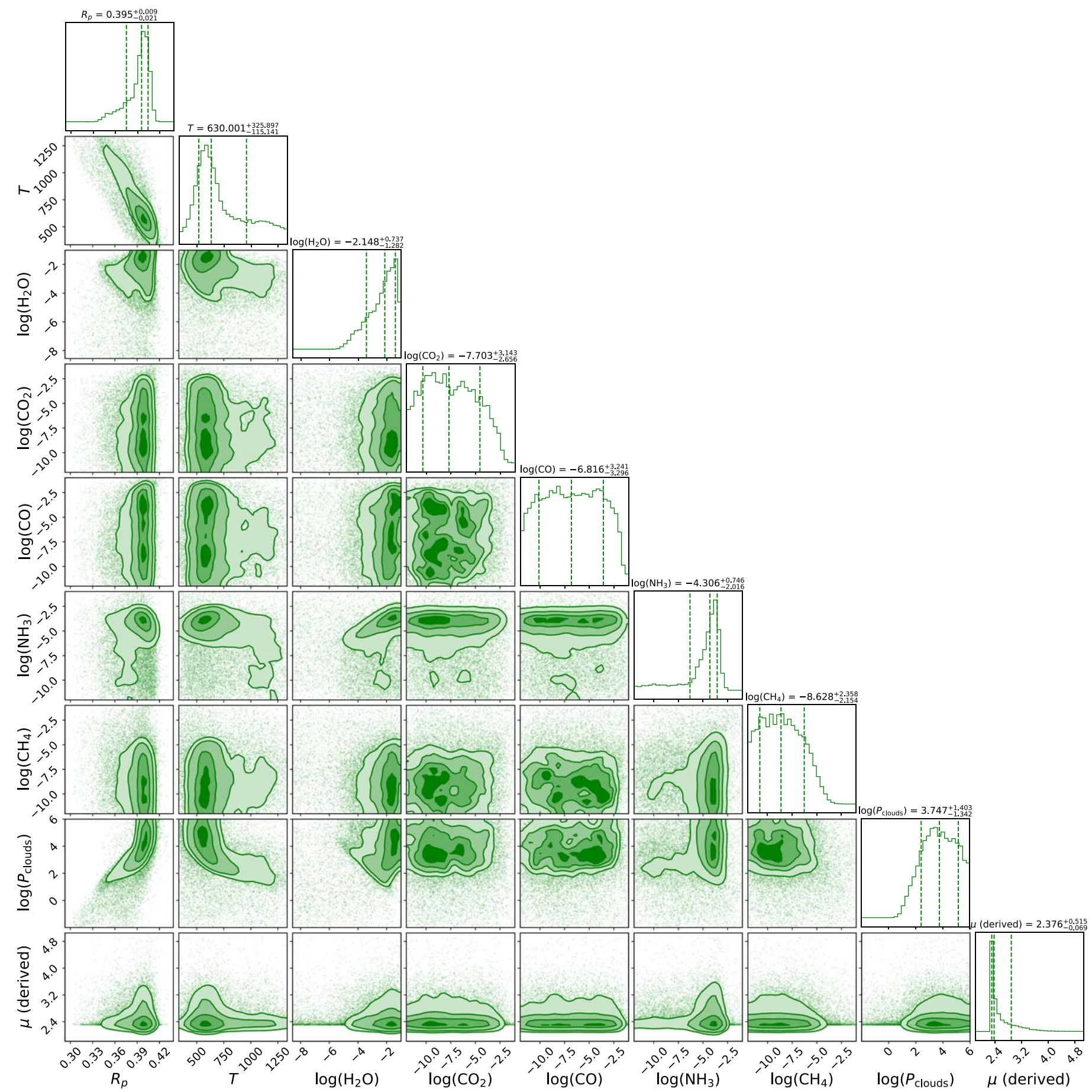

Figure 6. HD 106315c atmospheric retrieval posterior distributions.

abundances are unconstrained. The model finds a cloud's top pressure of $10^{3.7} \mathrm{~Pa}$ correlated to the amount of $\mathrm{H}_{2} \mathrm{O}$ : the deeper the clouds are, the more water we have. The best-fit solution suggests a clear atmosphere with a significant amount of water. In order to give an estimation of the planetary $\mathrm{C} / \mathrm{O}$ ratio, we employed the following formula readapted from MacDonald \& Madhusudhan (2019): $\mathrm{C} / \mathrm{O}=\left(\mathrm{XCH}_{4}+\right.$ $\left.\mathrm{XCO}+\mathrm{XCO}_{2}\right) /\left(\mathrm{XH}_{2} \mathrm{O}+\mathrm{XCO}+2 \mathrm{CO}_{2}\right)$, where the numerator indicates all species containing $\mathrm{C}$ atoms, while the denominator indicates all other O-bearing species. As we obtained a constrained value only for the water abundance, we decided to explore the range of valid $\mathrm{C} / \mathrm{O}$ by using not only the mean abundances but also the upper/lower possible values allowed by the posteriors (see Table 6). In this way, we obtained a $\mathrm{C} / \mathrm{O}$ ratio that could vary in the range of $7.5 \times 10^{-9}$ to 0.60 .

\subsection{2. $H D 3167 c$}

The ADI value found for HD 3167c retrieval is lower than the one computed for HD 106315c (Table 6), yet it corresponds 


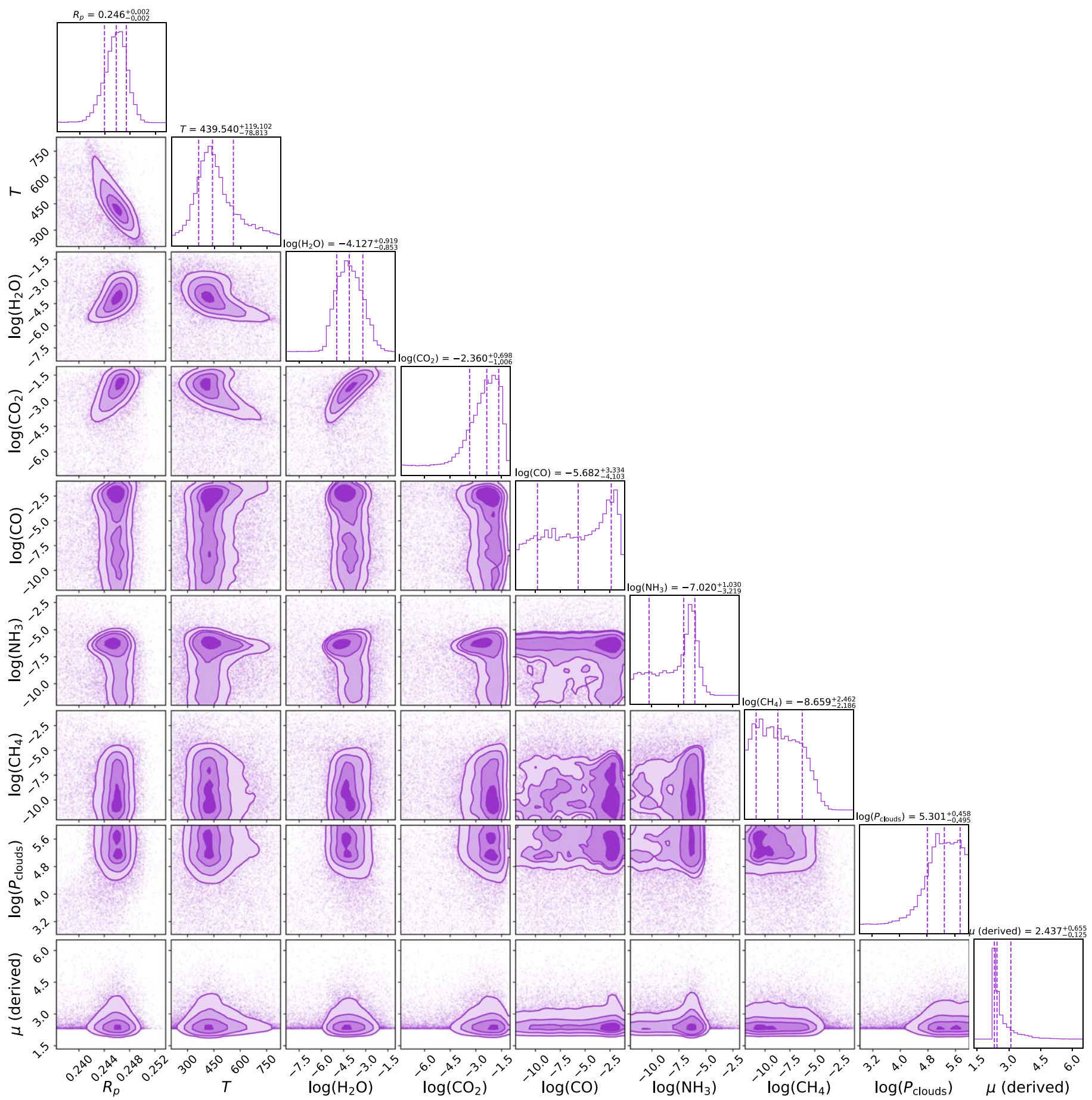

Figure 7. HD 3167c atmospheric retrieval posterior distributions.

to a $4.76 \sigma$ significance detection of an atmosphere around this sub-Neptune. The temperature retrieved by TauREx $\left(440_{-79}^{+119} \mathrm{~K}\right)$ is less than the equilibrium temperature obtained assuming an albedo equal to 0.2 , but it is consistent within $1 \sigma$.

The main difference with HD 106315c's atmosphere is the strong detection of $\mathrm{CO}_{2}$, and more generally the presence of carbon-bearing species. Opacity source contributions in Figure 5 show both water and carbon dioxide features; these two species seem required to fit the data obtained by HST/ WFC3, and their abundances are highly correlated (see Figure 7). $\Delta_{\mathrm{E} 2}$ is equal to 6.65 (Table 7), meaning that the full chemical model is statistically significant $(4.07 \sigma)$ compared to a pure water model. This is probably driven by the carbon dioxide detection that explains the absorption features at 1.20, 1.45 , and $1.60 \mu \mathrm{m}$. The best-fit solution contains a significant amount of carbon dioxide $\log _{10}\left[\mathrm{CO}_{2}\right]=-2.4_{-1.0}^{+0.7}$ and a lower amount of water $\log _{10}\left[\mathrm{H}_{2} \mathrm{O}\right]=-4.1_{-0.9}^{+0.9}$. As explained in Section 3.2, we would have expected $\mathrm{CH}_{4}$ to be the main carbon-bearing species instead of $\mathrm{CO}_{2}$.

Looking at the posterior distributions in Figure 7, we can constrain the higher limits of ammonia and methane abundances, which are below $10^{-5}$. The monoxide abundance 
Table 7

Comparison of the Bayesian Log Evidence for Different Models

\begin{tabular}{|c|c|c|c|c|c|c|c|c|c|c|c|}
\hline No. & Setup & $\log E$ & ADI & $\Delta_{\mathrm{E} 1}$ & $\Delta_{\mathrm{E} 2}$ & $T(\mathrm{~K})$ & $R_{\mathrm{P}}\left(R_{\mathrm{J}}\right)$ & $\log \left[P_{\text {clouds }} / 1 \mathrm{~Pa}\right]$ & $\log \left[\mathrm{H}_{2} \mathrm{O}\right]$ & $\log \left[\mathrm{NH}_{3}\right]$ & $\log \left[\mathrm{CH}_{4}\right]$ \\
\hline \multicolumn{12}{|c|}{ HD $106315 c$} \\
\hline $\mathrm{A} 0$ & No active gas & 210.94 & $\mathrm{~N} / \mathrm{A}$ & $\mathrm{N} / \mathrm{A}$ & $\mathrm{N} / \mathrm{A}$ & $798_{-315}^{+356}$ & $0.388_{-0.035}^{+0.027}$ & $2.5_{-3.2}^{+2.5}$ & $\mathrm{~N} / \mathrm{A}$ & $\mathrm{N} / \mathrm{A}$ & $\mathrm{N} / \mathrm{A}$ \\
\hline $\mathrm{A} 1$ & Full chemical & 226.91 & 15.97 & $\mathrm{~N} / \mathrm{A}$ & 6.07 & $630_{-115}^{+326}$ & $0.395_{-0.021}^{+0.009}$ & $3.7_{-1.3}^{+1.4}$ & $-2.1_{-1.3}^{+0.7}$ & $-4.3_{-2.0}^{+0.7}$ & $<-5$ \\
\hline $\mathrm{A} 2$ & $\mathrm{H}_{2} \mathrm{O}$ only & 220.84 & 9.52 & $\mathrm{~N} / \mathrm{A}$ & $\mathrm{N} / \mathrm{A}$ & $859_{-99}^{+66}$ & $0.404_{-0.002}^{+0.002}$ & $\mathrm{~N} / \mathrm{A}$ & $-5.1_{-0.2}^{+0.3}$ & $\mathrm{~N} / \mathrm{A}$ & $\mathrm{N} / \mathrm{A}$ \\
\hline $\mathrm{A} 3$ & No $\mathrm{H}_{2} \mathrm{O}$ & 212.70 & 1.76 & 14.21 & $\mathrm{~N} / \mathrm{A}$ & $417_{-56}^{+156}$ & $0.402_{-0.011}^{+0.006}$ & $4.1_{-1.8}^{+1.3}$ & $\mathrm{~N} / \mathrm{A}$ & $-3.4_{-1.5}^{+1.0}$ & $-3.0_{-2.9}^{+1.0}$ \\
\hline A4 & No clouds & 226.98 & 16.04 & $\mathrm{~N} / \mathrm{A}$ & 6.14 & $546_{-87}^{+93}$ & $0.402_{-0.007}^{+0.005}$ & $\mathrm{~N} / \mathrm{A}$ & $-2.1_{-1.5}^{+0.7}$ & $-4.3_{-1.0}^{+0.7}$ & $<-5$ \\
\hline A5 & No $\mathrm{NH}_{3}$ & 226.00 & 15.06 & 0.91 & 5.16 & $1004_{-278}^{+223}$ & $0.374_{-0.020}^{+0.022}$ & $2.5_{-0.9}^{+1.1}$ & $-2.6_{-1.3}^{+1.1}$ & $\mathrm{~N} / \mathrm{A}$ & $<-5$ \\
\hline \multicolumn{12}{|c|}{ HD 3167c } \\
\hline B0 & No active gas & 225.84 & $\mathrm{~N} / \mathrm{A}$ & $\mathrm{N} / \mathrm{A}$ & $\mathrm{N} / \mathrm{A}$ & $473_{-180}^{+225}$ & $0.238_{-0.016}^{+0.010}$ & $2.2_{-2.6}^{+2.6}$ & $\mathrm{~N} / \mathrm{A}$ & $\mathrm{N} / \mathrm{A}$ & $\mathrm{N} / \mathrm{A}$ \\
\hline B1 & Full chemical & 235.41 & 9.58 & $\mathrm{~N} / \mathrm{A}$ & 6.65 & $440_{-79}^{+119}$ & $0.246_{-0.002}^{+0.002}$ & $5.3_{-0.5}^{+0.5}$ & $-4.1_{-0.9}^{+0.9}$ & $-2.4_{-1.0}^{+0.7}$ & unconstrained \\
\hline $\mathrm{B} 2$ & $\mathrm{H}_{2} \mathrm{O}$ only & 228.76 & 2.92 & $\mathrm{~N} / \mathrm{A}$ & $\mathrm{N} / \mathrm{A}$ & $785_{-73}^{+33}$ & $0.2425_{-0.0006}^{+0.0009}$ & $\mathrm{~N} / \mathrm{A}$ & $-5.62_{-0.18}^{+0.19}$ & $\mathrm{~N} / \mathrm{A}$ & $\mathrm{N} / \mathrm{A}$ \\
\hline B3 & $\mathrm{No}_{2} \mathrm{O}$ & 231.80 & 5.97 & 3.61 & $\mathrm{~N} / \mathrm{A}$ & $449_{-88}^{+100}$ & $0.246_{-0.002}^{+0.002}$ & $5.1_{-0.6}^{+0.6}$ & $\mathrm{~N} / \mathrm{A}$ & $-1.9_{-0.8}^{+0.5}$ & unconstrained \\
\hline B4 & No clouds & 236.45 & 10.62 & $\mathrm{~N} / \mathrm{A}$ & 7.69 & $426_{-75}^{+127}$ & $0.246_{-0.002}^{+0.002}$ & $\mathrm{~N} / \mathrm{A}$ & $-4.2_{-0.8}^{+0.9}$ & $-2.4_{-1.0}^{+0.7}$ & unconstrained \\
\hline B5 & $\mathrm{No} \mathrm{CO}_{2}$ & 231.48 & 5.64 & 3.93 & 2.72 & $605_{-237}^{+151}$ & $0.245_{-0.003}^{+0.003}$ & $5.2_{-0.7}^{+0.5}$ & $-4.8_{-0.6}^{+1.4}$ & $\mathrm{~N} / \mathrm{A}$ & $-1.9_{-1.6}^{+0.5}$ \\
\hline B6 & No $\mathrm{CO}$ & 234.84 & 9.00 & 0.60 & 6.08 & $440_{-82}^{+110}$ & $0.246_{-0.002}^{+0.002}$ & $5.3_{-0.6}^{+0.5}$ & $-3.9_{-1.0}^{+1.1}$ & $-2.1_{-0.9}^{+0.7}$ & $\mathrm{~N} / \mathrm{A}$ \\
\hline B7 & $\mathrm{No} \mathrm{CO}_{2}, \mathrm{CO}$ & 229.86 & 4.03 & 5.55 & 1.10 & $732_{-105}^{+60}$ & $0.2423_{-0.0009}^{+0.0013}$ & $5.4_{-0.4}^{+0.4}$ & $-5.5_{-0.2}^{+0.3}$ & $\mathrm{~N} / \mathrm{A}$ & $\mathrm{N} / \mathrm{A}$ \\
\hline
\end{tabular}

Note. The logarithm is taken to the base-10 $\left(\log \rightarrow \log _{10}\right)$.

posterior distribution is highly degenerate, hence the weak detection. Carbon dioxide and monoxide features are difficult to distinguish in WFC3/G141 observations because they have similar features between 1.5 and $1.6 \mu \mathrm{m}$, leading potentially to degeneracies between the two abundances. The amounts of $\mathrm{H}_{2} \mathrm{O}$ and $\mathrm{CO}_{2}$, as well as the planet temperature and radius, are correlated. For less water and carbon dioxide, the model requires a higher temperature and lower radius at $10 \mathrm{bar}$ atmospheric pressure (see Figure 7). The best-fit solution suggests a clear atmosphere with a top cloud pressure retrieved at 1 bar. As for HD 106315c, we derived a range of possible values in which the $\mathrm{C} / \mathrm{O}$ ratio could vary, i.e., $0.49-0.85$.

\section{Discussion}

Considering the narrow wavelength coverage and the low data resolution, the results obtained here are to be considered carefully and put into perspective. The model we tested has eight free parameters and 25 observation data points. Molecular abundances and temperatures retrieved by TauREx are sensitive to the inputs and bounds set up by the users. TauREx gives us a first insight into these exoplanets' atmospheres and, in particular for HST/WFC3, helps us to infer the presence of water. We then analyzed different simulations (A0-A5 and B0-B7 in Table 7, for HD 106315c and HD 3167c, respectively) that include the expected molecules considering the wavelength coverage and the equilibrium temperature. We performed this operation to better constrain the molecular detections found in Section 3.2. A0 and B0 are flat-line models that help us compute the ADI and A2 and B2, pure water models are used to compute $\Delta_{\mathrm{E} 2}$.

\subsection{Strength of $\mathrm{H}_{2} \mathrm{O}$ Detection}

For both planets, to assess the significance of $\mathrm{H}_{2} \mathrm{O}$ detection, we removed this active gas from the full chemical model and analyzed the Bayes factor $\Delta_{\mathrm{E} 1}$. It decreases from 226.91 (A1) to 212.70 (A3) (see Table 7) and from 235.41 (B1) to 231.80 (B3) for $\mathrm{HD} 106315 \mathrm{c}$ and $\mathrm{HD} 3167 \mathrm{c}$, respectively. $\mathrm{H}_{2} \mathrm{O}$ detection is statistically confirmed for both planets with a "strong" significance $(5.68 \sigma)$ for HD $106315 \mathrm{c}$ and a "moderate" one $(3.17 \sigma)$ for HD 3167c. In the recent paper by Kreidberg et al. (2020), they reported a tentative detection (with a Bayes factor of 1.7 or 2.6, depending on prior assumptions) of water vapor with a small amplitude of $30 \mathrm{ppm}$. In this simultaneous and independent analysis, by using different algorithms both for the extraction of the transmission spectrum from the WFC3 data (with Iraclis) and for the retrieval analysis (performed with TauREx3), we also detect the presence of water in the atmosphere of HD 106315c with a high significance. Moreover, our observed spectrum seems to be compatible with deeper $\mathrm{H}_{2} \mathrm{O}$ features, which reinforces the detection. To date, water has been detected on several Neptune and sub-Neptune planets, which allows comparisons. HD $106315 \mathrm{c}$ could be compared to HAT-P-11 b (with a water detection's significance, hereafter $\sigma_{\mathrm{H}_{2} \mathrm{O}}$, of $5.1 \sigma$; Fraine et al. $2014)$ and to GJ 3470b $\left(\sigma_{\mathrm{H}_{2} \mathrm{O}}=5.2 \sigma\right.$; Benneke et al. 2019a). While HD 3167c has a lower water detection, appearing more similar to $\mathrm{K} 2-18 \mathrm{~b} \quad\left(\sigma_{\mathrm{H}_{2} \mathrm{O}}=3.6 \sigma\right.$, Tsiaras et al. 2019; $\sigma_{\mathrm{H}_{2} \mathrm{O}}=3.93 \sigma$, Benneke et al. 2019b). Crossfield \& Kreidberg (2017) studied the water feature amplitude of six warm Neptune planets and highlighted correlations with the equilibrium temperature and the mass fraction of hydrogen and helium. To verify the correlation of $\mathrm{H}_{2} \mathrm{O}$ amplitude, in units of atmospheric scale height, with the equilibrium temperature, we computed the water amplitude for HD 106315c, HD 3167c, and K2-18b using HST/WFC3 spectra obtained here and in Tsiaras et al. (2019). We used the same method described in Crossfield \& Kreidberg (2017). We fitted a carbon-free template of GJ 1214b normalized in units of scale height (Crossfield et al. 2011) to the observations using the Levenberg and Marquardts least-squares method (L-M; Markwardt 2009). Then, we measured the amplitude by taking the normalized average value from 1.34 to $1.49 \mu \mathrm{m}$ and subtracting it from the average value outside this wavelength range. The scale height $H=K_{\mathrm{B}} T_{\text {eq }} / \mu \mathrm{g}$ is computed assuming a hydrogen-rich atmosphere $(\mu=2.3 \mathrm{amu})$, and the equilibrium temperature is calculated for an albedo of 0.2 . We find a water feature amplitude of $1.02 \pm 0.18$ for HD 106315c, $1.04 \pm 0.24$ for 


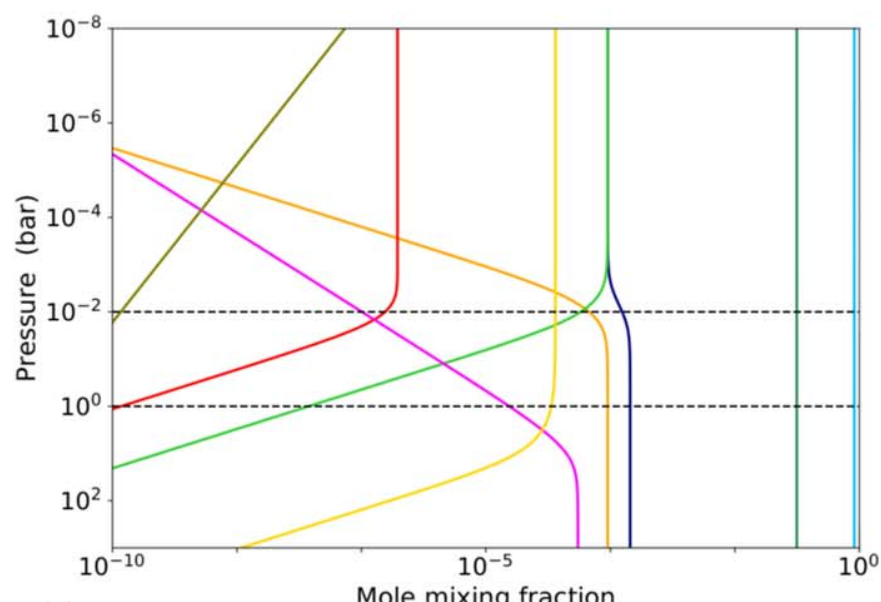

(a)

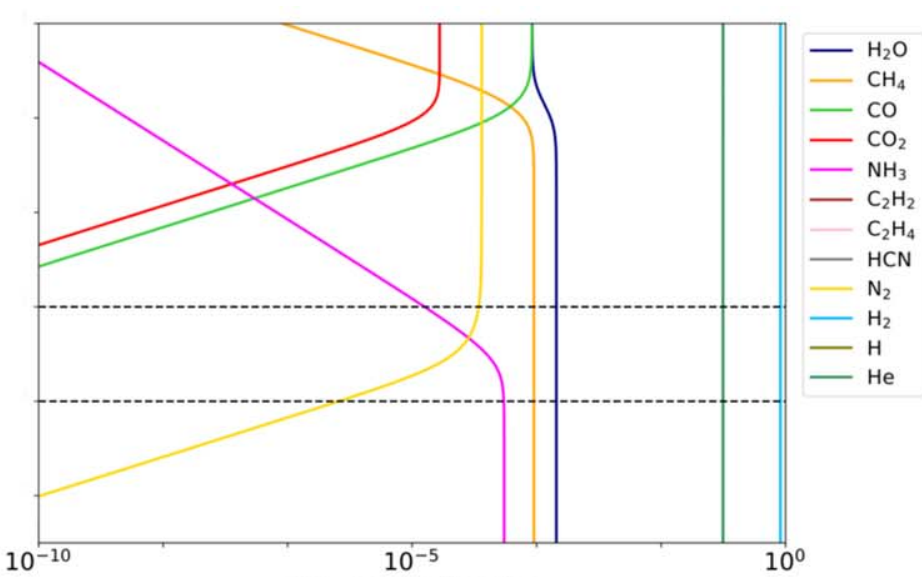

(b)

Mole mixing fraction

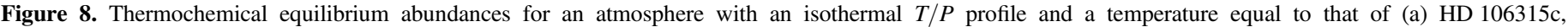

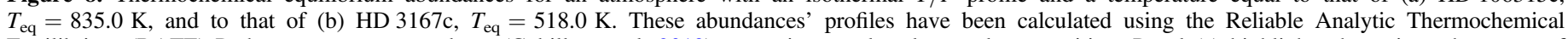

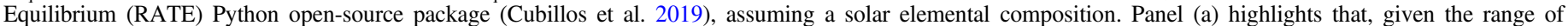

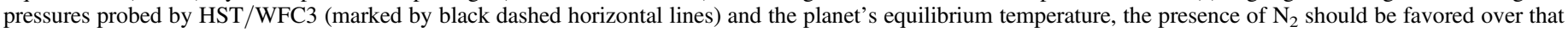
of $\mathrm{NH}_{3}$ in the atmosphere of $\mathrm{HD} 106315 \mathrm{c}$. Moreover, panel (b) shows that $\mathrm{H}_{2} \mathrm{O}, \mathrm{CH}_{4}$, and $\mathrm{NH}_{3}$ are the expected molecules in the atmosphere of $\mathrm{HD} 3167 \mathrm{c}$.

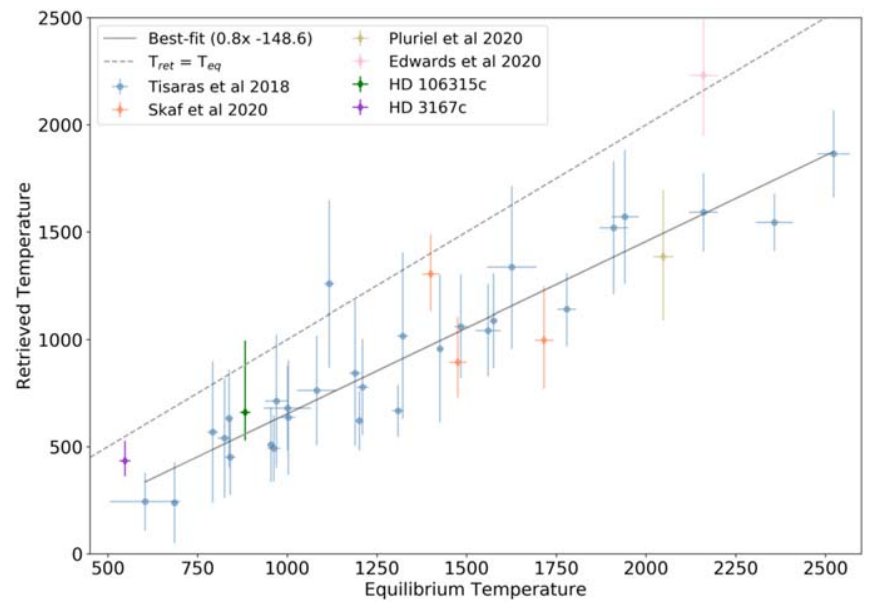

Figure 9. Trend between the retrieved and the equilibrium temperatures (or irradiation temperature) for the planets studied in Tsiaras et al. (2016c), those studied in Skaf et al. (2020), and the two planets analyzed in this work. For consistency with the above-mentioned two works, a zero albedo has been assumed to calculate the equilibrium temperature. For completeness, the planets studied in ARES I (Edwards et al. 2020) and in ARES III (Pluriel et al. 2020a) are shown too.

HD 3167c, and $1.28 \pm 0.49$ for K2-18b. We note that Kreidberg et al. (2020) recently found a lower absorption feature, i.e., $0.80 \pm 0.04$ for HD $106315 \mathrm{c}$. We plot our values in Figure 10 along with the amplitudes computed in Crossfield \& Kreidberg (2017) and the ones found in Libby-Roberts et al. (2020) for Kepler 51 b and Kepler 51 d. Finally, we fitted a linear relation and compared the Pearson correlation coefficient and the probability. We find a correlation coefficient of 0.43 and a $p$-value of 0.18 . The strong correlation highlighted in Crossfield \& Kreidberg (2017) is not found here, mostly because of K2-18b's high water feature amplitude at low temperature. While removing K2-18b and Kepler $51 \mathrm{~d}$ amplitudes to focus on planets with temperature between 500 and $1000 \mathrm{~K}$ as in Crossfield \& Kreidberg (2017), we find a correlation coefficient of 0.70 and $p$-value of 0.04 , while they found a coefficient of 0.83 for a $p$-value equal to 0.04 . A refinement of the scale height, HST/WFC3 water amplitude, and correlation computations will be detailed in a follow-up paper focusing on intermediate-size planets $\left(R_{\mathrm{P}}<6 R_{\oplus}\right)$ with consistent published spectra.

\subsection{Clear or Cloudy Atmospheres}

In Section 3.2, we retrieved a clear atmosphere for both planets, but we expect species to condense and clouds to form on warm Neptune and sub-Neptune planets. The flat spectra of GJ 436b (Knutson et al. 2014a), GJ 1214b (Kreidberg et al. 2014a), and HD 97658b (Knutson et al. 2014b) were interpreted as high cloud or haze at low pressure. We confirm the clear atmosphere by removing the cloud top pressure parameter from the full chemical model. ADIs of cloud-free models (A4 and B4 in Table 7) are higher than ADIs of full chemical models including clouds (A1 and B1 in Table 7). Clouds do not impact retrieval results, even for HD $106315 \mathrm{c}$ with a lower top clouds pressure, and this means that either the planet has a clear atmosphere or the clouds are located below the visible pressure where the atmosphere is opaque. Looking at HD $106315 c$ 's cloud top pressure correlations with $\mathrm{H}_{2} \mathrm{O}$ abundance (see Figure 6), a second mode appears, meaning that clouds could be present in the region we are probing.

The TauREx retrieval does not bring any information on cloud composition, and we must recall that the wavelength coverage is not wide enough to constrain cloud chemistry. All things considered, models have predicted that for hot atmospheres (900 to $1300 \mathrm{~K}$ ) we could find condensates like $\mathrm{KCl}$, $\mathrm{ZnS}$, and $\mathrm{Na}_{2} \mathrm{~S}$, and for colder atmospheres (400 to $\left.600 \mathrm{~K}\right) \mathrm{KCl}$ and $\mathrm{NH}_{4} \mathrm{H}_{2} \mathrm{PO}_{4}$ (Lodders \& Fegley 2006; Morley et al. 2012). GJ 1214b (6.26 $\pm 0.86 M_{\oplus}, 2.85 \pm 0.20 R_{\oplus}$; Harpsøe et al. 2013), K2-18b (8.92 $\pm 1.7 M_{\oplus}, 2.37 \pm 0.22 R_{\oplus}$; Sarkis et al. 2018), and HD 3167c (this paper) have a similar mass and radius, and yet they present very different atmospheric properties. The equilibrium temperature is lower for $\mathrm{K} 2-18 \mathrm{~b}$ $(284 \pm 15 \mathrm{~K}$; Sarkis et al. 2018) but presents water detection. GJ $1214 \mathrm{~b}$ has a similar equilibrium temperature $\left(547_{-8}^{+7}\right.$; Kundurthy et al. 2011) but exhibits a flat spectrum suggesting the presence of clouds. 


\section{3. $\mathrm{NH}_{3}$ in $\mathrm{HD} 106315 \mathrm{c}$ 's Atmosphere}

HD 106315c's best-fit solution includes a small amount of $\mathrm{NH} 3$, i.e., $\log _{10}\left[\mathrm{NH}_{3}\right]=-4.3_{-2.0}^{+0.7}$. Looking at the posterior distribution (Figure 6), $\mathrm{NH}_{3}$ abundance converges toward a solution. To confirm this detection, we removed this gas from the full chemical model and computed $\Delta_{\mathrm{E} 1}$ (see A5 in Table 7). The difference is 0.91 , meaning that $\mathrm{NH}_{3}$ 's detection has to be considered "not significant" $(1.97 \sigma)$. However, we observe some differences: the temperature rises to $1004 \mathrm{~K}$ with fewer constraints, and consequently, the radius decreases to $0.374 R_{\mathrm{J}}$. Clouds are found at a higher level of $10^{2.5} \mathrm{~Pa}$. The cloud deck compensates for $\mathrm{NH}_{3}$ features by cutting $\mathrm{H}_{2} \mathrm{O}$ ones and shrinking the spectrum. From this analysis, we conclude that HD 106315 c can be surrounded by either a primary clear atmosphere with $\mathrm{H}_{2} \mathrm{O}$ and traces of $\mathrm{NH}_{3}$ or a primary atmosphere with $\mathrm{H}_{2} \mathrm{O}$ and deep clouds.

As mentioned in Section 3.1, the high equilibrium temperature of HD 106315c should have favored the presence of $\mathrm{N}_{2}$ instead of $\mathrm{NH}_{3}$ (see, e.g., Figure 8). $\mathrm{NH}_{3}$ is expected to disappear above $500-550 \mathrm{~K}$. However, we retrieve at the terminator, so we should expect a lower temperature (closer to this $500 \mathrm{~K}$ limit) and more $\mathrm{NH}_{3}$. Moreover, $\mathrm{N}_{2}$ is an inactive gas, with no feature in WFC3, which means that the free retrieval we perform - the retrieval in free mode is used to retrieve the abundance for active molecules that have features in the spectrum-will not pick up this molecule except if it influences the mean molecular weight. To test this, we added $\mathrm{N}_{2}$ in the analysis to see the possible consequences that this molecule could have had on the mean molecular weight. We assumed an initial $\mathrm{N}_{2}$ abundance of $10^{-4}$, compatible with the one expected by thermochemical equilibrium conditions (see Figure 8), and we allowed it to vary between $10^{-12}$ and $10^{-1}$ in volume mixing ratios (log-uniform prior), as for the other molecules. The inclusion of $\mathrm{N}_{2}$ does not affect the mean molecular weight, a simple cloud model added to $\mathrm{H}_{2} \mathrm{O}$ and $\mathrm{NH}_{3}$ features is enough to fit the spectrum, and there is no need to add extra molecular weight to shrink the spectrum. Moreover, $\mathrm{NH}_{3}$ detection remains around $10^{-4}$ (see Figure A3).

\section{4. $\mathrm{CO}_{2}$ in $\mathrm{HD} 3167 c$ 's Atmosphere}

HD 3167c best-fit solution includes an important amount of $\mathrm{CO}_{2}$ (i.e., $\log _{10}\left[\mathrm{CO}_{2}\right]=-2.4_{-1.0}^{+0.7}$ ). This detection is supported by the data points from $\sim 1.5$ to $1.6 \mu \mathrm{m}$, but water seems to explains better the absorption features around $1.4 \mu \mathrm{m}$ (see Figure 5). We removed $\mathrm{CO}_{2}$ from the full chemical and compared log evidences; it decreases from 235.41 (B1, Table 7) to 231.48 (B5), corresponding to a $3.28 \sigma$ "moderate" detection. The ADI decreases as well to 5.64. We note that $\mathrm{CO}$ is now compensating for $\mathrm{CO}_{2}$ features and its $\log$ abundance increases to $\log _{10}[\mathrm{CO}]=-1.9_{-1.6}^{+0.5}$. This value is too high for a realistic primary hydrogen-rich atmosphere that we expect for this planet. We successively removed $\mathrm{CO}$ from the full chemical model, but it does not impact the retrieval results (B6 in Table 7), and $\Delta_{\mathrm{E} 1}$ is below 1 ("not significant"). Finally, we removed both $\mathrm{CO}$ and $\mathrm{CO}_{2}$ to assess the detection of those carbon-bearing species (B7). The difference in log evidences is now equal to $\Delta_{\mathrm{E} 1}=5.55$ and corresponds to more than $3 \sigma$ carbon detection. This test does not impact the abundance of water or the top cloud pressure, but constrains better the abundance of ammonia to $10^{-6.4}$. We note that $\mathrm{CH}_{4}$ does not compensate the lack of the other carbon-bearing species; its abundance remains constrained below $10^{-5}$. The temperature increases to keep a primary atmosphere hypothesis and an extended clear atmosphere.

This unexpected detection of carbon-bearing species could be explained by noise or systematic effects that were not removed during the white-light-curve fitting step (see Section 2.4). It could also be the result of phenomena that our $1 \mathrm{D}$ equilibrium chemistry modeling cannot reproduce, e.g., 3D transport crossterminator. Another interpretation could be the actual presence of $\mathrm{CO}_{2}$ in the atmosphere of HD 3167c due, for example, to a very high metallicity, enhanced over that of the host star, which is consistent with solar metallicity $([\mathrm{Fe} / \mathrm{H}]=0.03 \pm 0.03 \mathrm{dex}$; Gandolfi et al. 2017). It is indeed known that the abundance of $\mathrm{CO}_{2}$ scales quadratically with metallicity (see, e.g., Moses 2014), and other examples of overabundance of $\mathrm{CO}_{2}$ interpreted as caused by a high metallicity can be found in the literature (see, e.g., Madhusudhan \& Seager 2011). However, if we use the water abundance as a proxy of metallicity (see, e.g., Kreidberg et al. 2014b), we infer a solar or subsolar metallicity for HD $3167 \mathrm{c}$, which would be in tension with the possibility that $\mathrm{CO}_{2}$ could be present owing to high metallicity. More observations are thus necessary to better constrain the possible presence of $\mathrm{CO}_{2}$ in the atmosphere of HD 3167c.

\subsection{Inferences from the Mass and Radius}

There is a strong degeneracy in exoplanet interiors, as there are many compositional models that are compatible with an observed mass and radius. However, by combining the mass, radius, and spectroscopic results of our study, we can get an inference for the interior composition of $\mathrm{HD} 106315 \mathrm{c}$ and $\mathrm{HD} 3167$ c. Our discovery of icy constituents, such as $\mathrm{H}_{2} \mathrm{O}$ in both planetary atmospheres (and maybe $\mathrm{NH}_{3}$ in the envelope of HD 106315c), indicates an ice-rich embryo. Curiously, the mass and radius of HD 106315c and HD 3167c are also consistent with an ice-rich core, which we explain below.

For the following results we adopted the planetary models from Zeng \& Sasselov (2013), Zeng et al. (2016), and Zeng et al. (2019). Based on the mass and radius of HD 106315c and HD 3167c, they are both consistent with icy cores with hydrogen envelopes $\sim 5 \mathrm{wt} \%$ and $0.3-1 \mathrm{wt} \%$ of their total planetary masses, respectively. We show these results in Figure 11. Nevertheless, there is still enough uncertainty in the results that a silicate embryo engulfed by a hydrogen atmosphere is still plausible for both planets. Certainly, with improved mass and radius measurements, together with more accurate spectroscopic observations, the interior structure of exoplanets such as HD 106315c and HD 3167c will get further constrained. We discuss the implications of this in Section 4.7.

Besides, Mousis et al. (2020) recently showed that close-in planets could have water-rich hydrospheres in supercritical state. Their model suggests that intermediate-size planets could be hydrogen/helium-free and their interiors would simply vary from one another depending on the water content.

\subsection{Comparison with Previous Results}

This paper is the result of work carried out during the ARES Summer School, where we used algorithms and data available to the public, thus allowing our results to be tested and reproduced. This is the fourth paper output of this summer school. In the first work, ARES I (Edwards et al. 2020), and in 


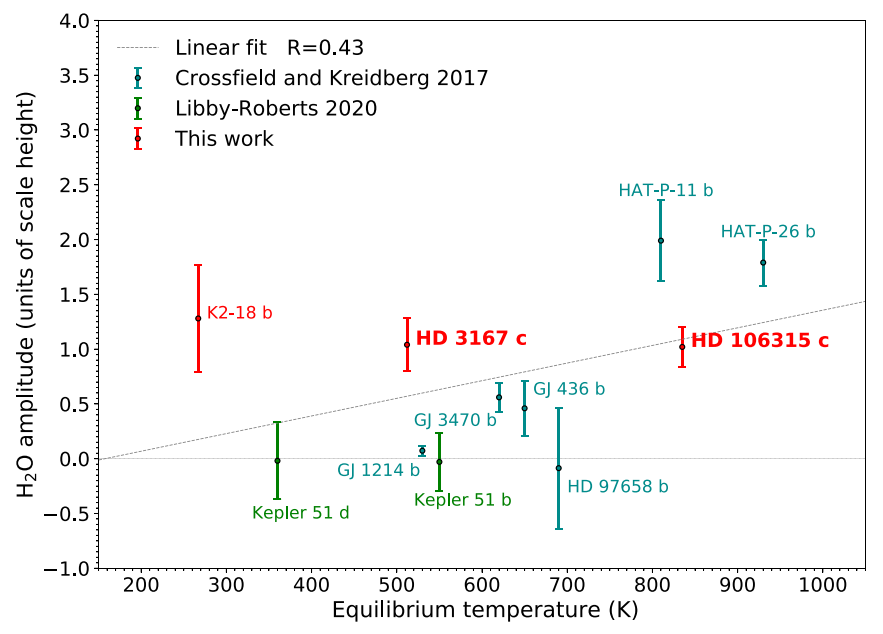

Figure 10. Normalized $\mathrm{H}_{2} \mathrm{O}$ amplitude in units of scale height with respect to equilibrium temperatures. Blue points are from Crossfield \& Kreidberg (2017), and green points are from Libby-Roberts et al. (2020). Red points are computed using the method described in Crossfield \& Kreidberg (2017) and spectra obtained in this work and from Tsiaras et al. (2019) for K2-18b. The dotted line corresponds to a linear fit. The correlation coefficient was found to be lower than in Crossfield \& Kreidberg (2017), 0.43 compared to 0.83 .

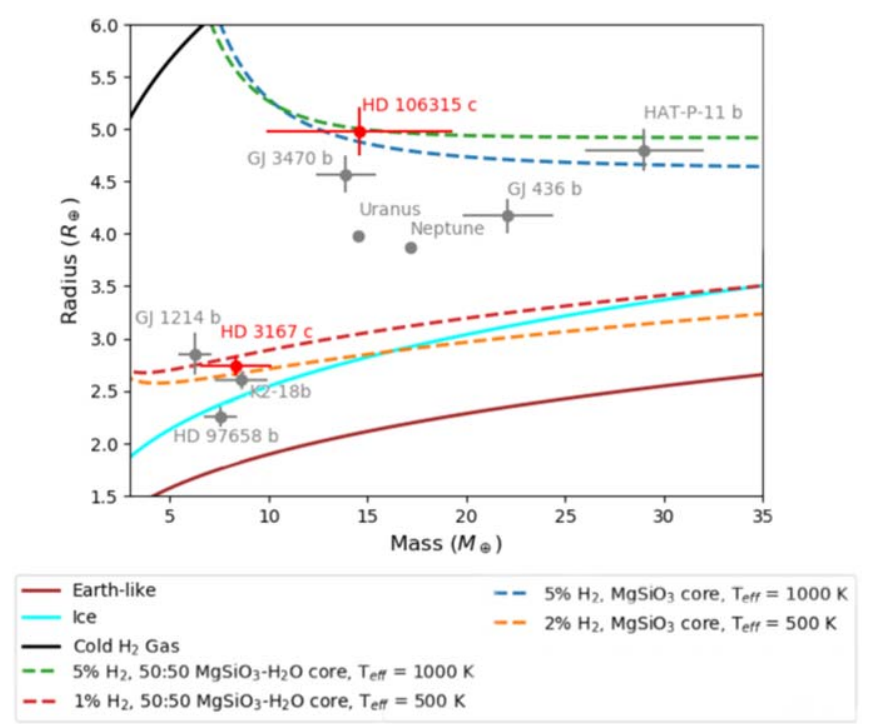

Figure 11. Mass and radius of HD 106315c and HD 3167c (from Table 2) plotted against other planets with size between 1.5 and $4 R_{\oplus}$ and published atmospheric characterization studies (see Table 1) — GJ 3470b (Awiphan et al. 2016), GJ 436 b (Maciejewski et al. 2014), GJ 1214b (Harpsøe et al. 2013), HD 97658b (van Grootel et al. 2014), HAT-P-11 b (Stassun et al. 2017), K2-18b (Benneke et al. 2019b) — and Uranus and Neptune (https://nssdc.gsfc.nasa.gov/planetary/ factsheet/). The mass and radius models are from Zeng \& Sasselov (2013) and Zeng et al. (2016).

the third one, ARES III (Pluriel et al. 2020a), we analyzed the transmission and the emission spectra of WASP-76b and Kelt$7 \mathrm{~b}$, respectively, while in the second one, ARES II (Skaf et al. 2020), the atmospheric study of WASP-42b, WASP-79b, and WASP-127b was performed. In this work, we used the ADI as a significance index to make the approach in our work uniform with these previous papers, with Tsiaras et al. (2018), and with Tsiaras et al. (2019). Figure 12 shows the gaseous exoplanets studied by Tsiaras et al. (2018) (in black); K2-18b examined in Tsiaras et al. (2019) (in blue); the hot Jupiters analyzed in ARES I, ARES II, and ARES III (in red)_for consistency with other works here we plot the ADI obtained from the analysis of WASP-76b's and Kelt-7 b's transmission spectra-and finally the Neptune-like planets, HD 106315c (in green) and HD 3167c (in violet), studied in this paper. From this figure, it emerges that, even if the two exoplanets characterized in this paper have smaller radii than most of the other targets, their ADI is not smaller. Our study, together with Tsiaras et al. (2019), shows that even smaller planets' atmospheres can be characterized with high significance. This opens the way for the atmospheric study of planets with smaller radii than the hot Jupiter targets that have mostly been analyzed so far.

\subsection{Future Characterization}

It is evident that in the future the exoplanetary field will be based on the detailed characterization of exo-atmospheres. In this scenario, NASA's upcoming JWST will play an important role; its large aperture, high sensitivity, and wide spectral range will allow the detection of molecular species in the atmospheres of planets with different masses: from super-Earths to super-Jovians. Scheduled to launch in the late 2020s, the ESA ARIEL space mission will enable atmospheric characterization of a large sample $(\sim 1000)$ of exoplanets in order to address how the chemical composition of an exoplanet is linked to its formation/evolution environment (Tinetti et al. 2018; Edwards et al. 2019a). With this prospect in mind, HD 106315c and HD 3167c represent suitable targets for both these space-borne instruments, and so we used the ARIEL Radiometric Model (ARIELRad) (Mugnai et al. 2020) to simulate observations by ARIEL. For each planet, we took the best-fit solution from the HST/WFC3 analysis to model ARIEL observations at its native resolution (i.e., the TIER 3 resolution); we considered 10 ARIEL transits. In addition, we simulated JWST observations using ExoWebb (B. Edwards et al. 2020, in preparation), assuming the collection of one single transit using NIRISS GR700XD plus a transit with NIRSpec G395M. Figure 13 shows, for the two planets, the results of our simulations for both JWST (left panels, (a) and (c)) and ARIEL (right panels, (b) and (d)). It highlights the increased wavelength coverage and data quality that will be obtained with both ARIEL and JWST. The power of having a broad wavelength coverage is that we can probe multiple absorption bands for each molecule. This helps break degeneracies due to overlapping features and always helps molecular compositions to be more readily constrained. Additionally, these future missions could shore up the detections of both $\mathrm{NH}_{3}$ (for $\mathrm{HD} 106315 \mathrm{c}$ ) and $\mathrm{CO}_{2}$ (for HD 3167c): the larger the spectral range covered, the more absorption bands may be present. Namely, on one hand, JWST and ARIEL could highlight the $\mathrm{CO}_{2}$ absorption features between $\sim 1.7-2.0 \mu \mathrm{m}$ and $4.0-5.32 \mu \mathrm{m}$; on the other hand, $\mathrm{NH}_{3}$ presents strong absorption features at longer wavelengths compared to the one probed with WFC3.

\section{Summary and Conclusion}

We presented here the analysis of HST/WFC3 spatially scanned observations of the Neptune-type HD 106315c and of the sub-Neptune HD 3167c, resulting in the detection of water vapor in both atmospheres. Starting from the raw data and using the routine Iraclis, we extracted a transmission spectrum for both planets. We then interpreted it through the use of the Bayesian spectral retrieval algorithm TauREx3. We found a statistically significant atmosphere surrounding the two 


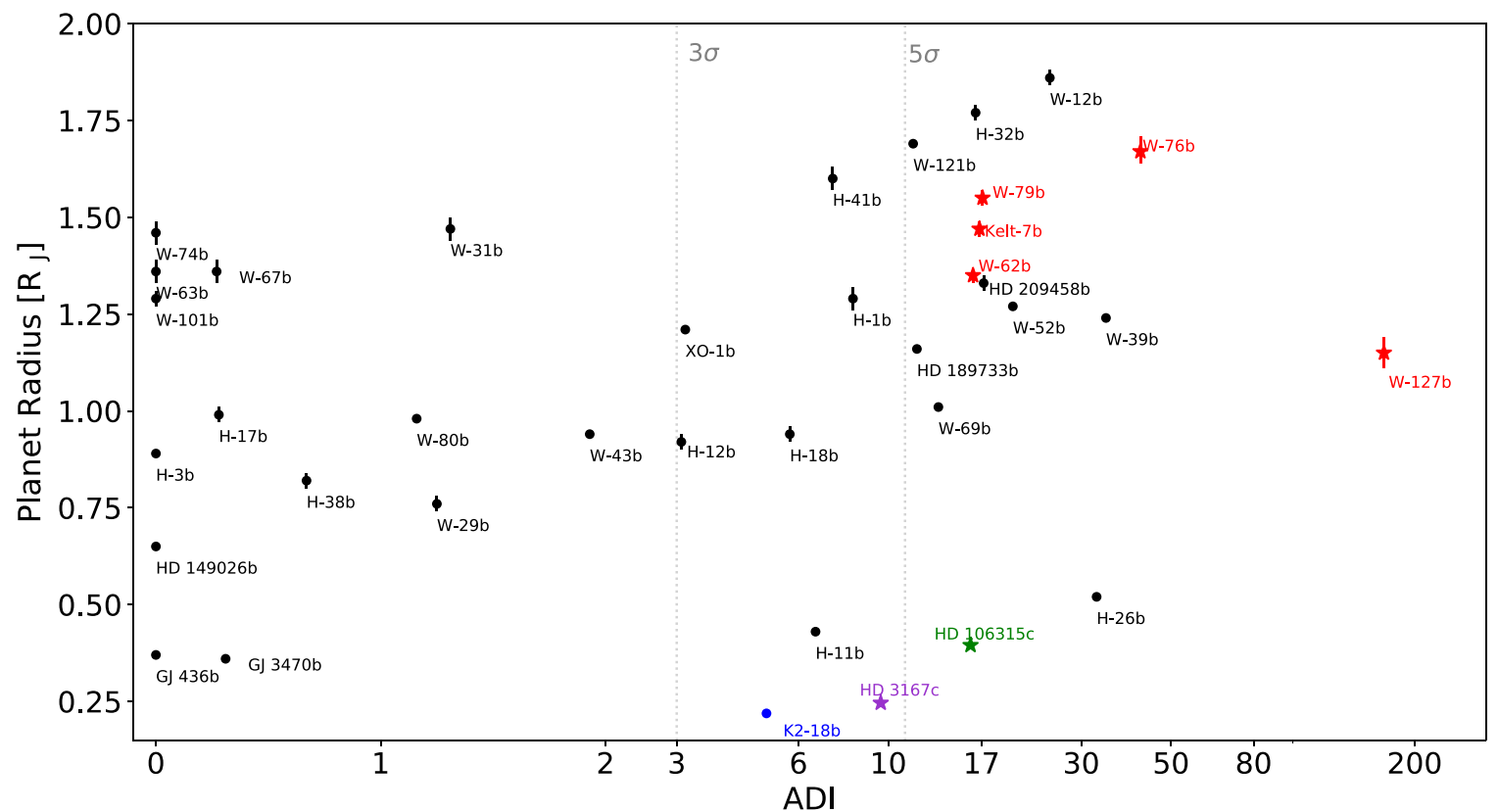

Figure 12. Exoplanetary radii as a function of the ADI index (in logarithmic scale) for the targets analyzed in this work (in green and in violet), in ARES I-III (in red), in Tsiaras et al. (2016c) (in black), and in Tsiaras et al. (2019) (in blue).

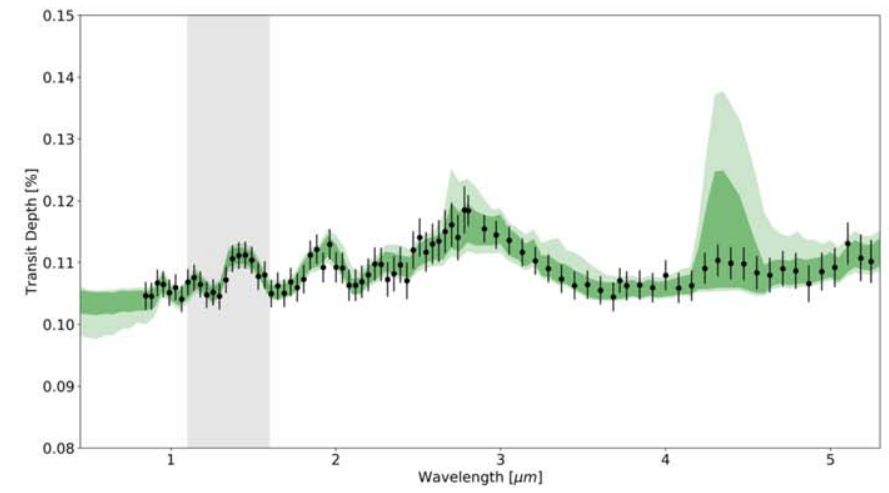

(a)

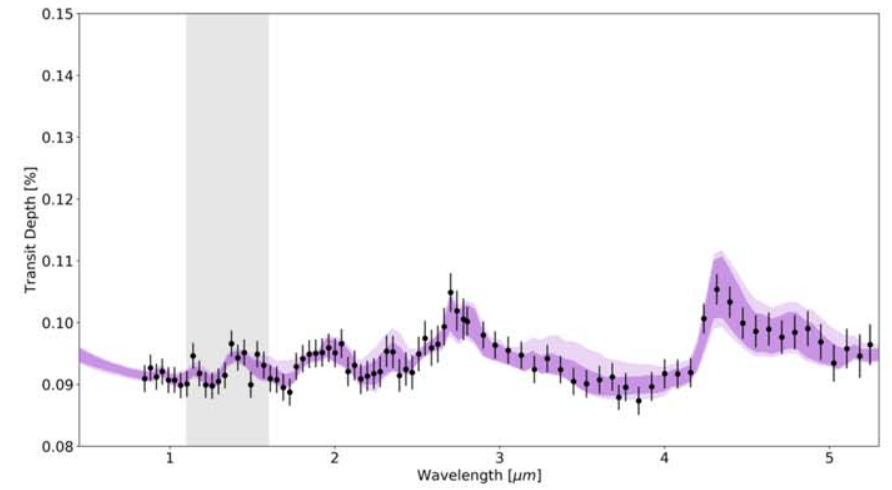

(c)

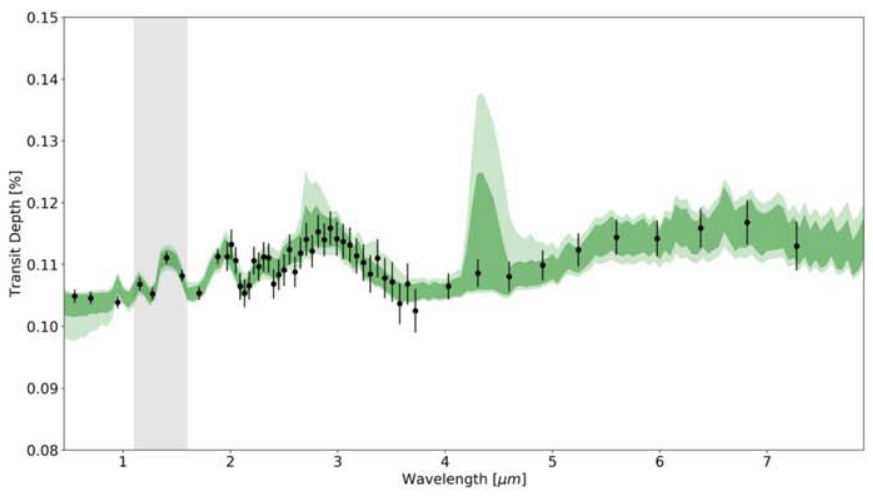

(b)

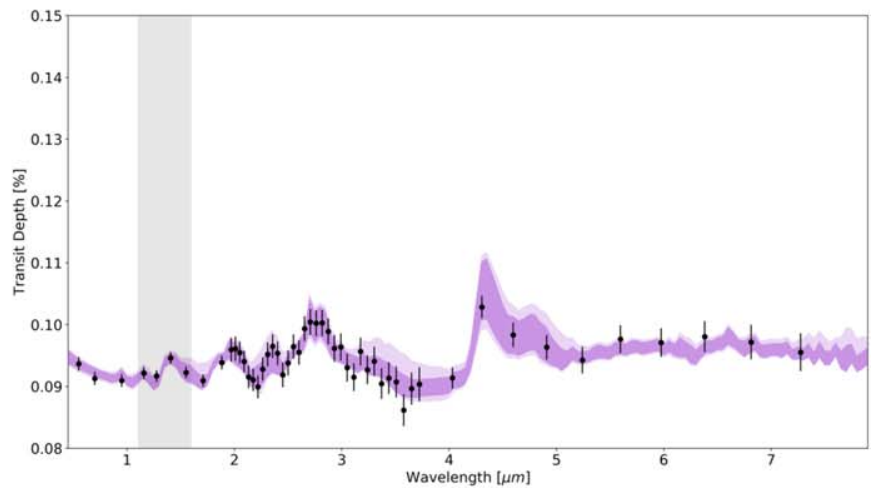

(d)

Figure 13. Simulated (a, c) JWST and (b, d) ARIEL observations of the best-fit solutions, i.e., the full chemical scenario, retrieved in this work. For ARIEL, 10 transits have been assumed for each planet, while JWST simulations have been performed using a single transit with NIRISS GR700XD, as well as an observation with NIRSpec G395H. HD 106315c and HD 3167c are shown in green (a and b) and in purple (c and d), respectively.

planets and evaluated the strength of our detection through the ADI metric.

From the TauREx analysis, we retrieved a "strong" detection of $\mathrm{H}_{2} \mathrm{O}\left(\log _{10}\left[\mathrm{H}_{2} \mathrm{O}\right]=-2.1_{-1.3}^{+0.7}, \Delta_{\mathrm{E} 1}=14.21\right)$ and "possible evidence" of $\mathrm{NH}_{3}\left(\log _{10}\left[\mathrm{NH}_{3}\right]=-4.3_{-2.0}^{+0.7}, \Delta_{\mathrm{E} 1}=0.91\right.$, even if it is not significant) in the atmosphere of HD 106315c. When removing ammonia, a deep cloud deck is required to fit the spectrum. We can only put an upper bound on methane 
abundance $\left(10^{-5}\right)$, while carbon dioxide and monoxide abundances are unconstrained.

The HD 3167c analysis resulted in both a water vapor $\left(\log _{10}\left[\mathrm{H}_{2} \mathrm{O}\right]=-4.1_{-0.9}^{+0.9}, \Delta_{\mathrm{E} 1}=3.61\right)$ and a carbon dioxide $\left(\log _{10}\left[\mathrm{CO}_{2}\right]=-2.4_{-1.0}^{+0.7}, \Delta_{\mathrm{E} 1}=3.93\right)$ "moderate" detection. As $\mathrm{CO}_{2}$ is not explained by $1 \mathrm{D}$ equilibrium chemistry models, its presence could be due to noise and highlights the limitations of our data quality. More precise constraints on the chemical abundances could be given if 3D models were employed instead of $1 \mathrm{D}$ ones. The shortcomings of retrieval analyses performed with 1D forward models have been highlighted already in previous papers (see, e.g., Caldas et al. 2019). On the contrary, if we assume a high metallicity, $\mathrm{CO}_{2}$ could actually be present in the atmosphere of HD 3167c (an increase in metallicity by a factor of $x$ tends to increase the abundance of $\mathrm{CO}_{2}$ by a factor of $x^{2}$; see, e.g., Moses 2014), and what we are seeing could not be due to noise or to systematics. Thus, further observations are needed to establish whether the $\mathrm{CO}_{2}$ might actually be present in the atmosphere of this exoplanet.

The future is bright for atmospheric studies of exoplanets thanks to both space-based and ground-based facilities. On one hand, Cowan et al. (2015), Greene et al. (2016), Tinetti et al. (2018), and Edwards et al. (2019b) have shown the potential of the JWST, Twinkle, and ARIEL space missions to characterize exo-atmospheres. On the other, ground-based instruments such as the European Extremely Large Telescope (E-ELT) - and in particular the Mid-Infrared E-ELT Imager and Spectrograph (METIS) instrument (Brandl et al. 2018) - the Thirty Meter Telescope (TMT; Skidmore et al. 2018), and the Giant Magellan Telescope (GMT; Fanson et al. 2018) will become available. This will lead to the systematic study of thousands of exoplanets' day sides and terminators both at high (HRS, from the ground) and at low (LRS, from space) spectral resolution. By combining HRS with LRS, and thus probing different regions of the exoplanetary atmospheres (higher atmospheric altitudes with HRS, lower atmospheric altitudes with LRS), we will better understand the atmospheric compositions and thus be able to apply more constraints on their formation and evolution. Given the brightness of their respective host stars and the large scale heights we computed $(H \sim 518 \pm 174 \mathrm{~km}$ and $H \sim 171 \pm 40 \mathrm{~km}$ for HD $106315 \mathrm{c}$ and HD 3167c, respectively), the two Neptune-like planets we studied in this paper are suitable targets for these upcoming instruments.

We want to thank the anonymous referee for the constructive comments that helped improve the quality of the manuscript.
This work was realized as part of "ARES ARIEL School" in Biarritz in 2019. The school was organized by J.-P.B., A.T., and I.W. with the financial support of CNES. J.-P.B. acknowledges the support of the University of Tasmania through the UTAS Foundation and the endowed Warren Chair in Astronomy, Rodolphe Cledassou, Pascale Danto and Michel Viso (CNES). W.P., T.Z., and A.Y.J. have received funding from the European Research Council (ERC) under the European Union's Horizon 2020 research and innovation program (grant agreement No. 679030/WHIPLASH and No. 758892/ExoAI). S.W. was supported through the STFC UCL CDT in Data Intensive Science (grant No. ST/P006736/1). G.G. acknowledges the financial support of the $2017 \mathrm{PhD}$ fellowship program of INAF. R.B. is a PhD fellow of the Research Foundation-Flanders (FWO). D.B. acknowledges financial support from the ANR project "e-PYTHEAS" (ANR16-CE31-0005-01). L.V.M. and D.M.-G. acknowledge the financial support of the ARIEL ASI grant No. 2018-22-HH.0. B.E., Q.C., M.M., A.T., and I.W. acknowledge funding from the European Research Council (ERC) under the European Union's Horizon 2020 research and innovation program grant ExoAI (GA No. 758892) and the STFC grants ST/P000282/1, ST/P002153/1, ST/S002634/1, and ST/T001836/1. N.S. acknowledges the support of the IRIS-OCAV, PSL. M.P. acknowledges support by the European Research Council under grant agreement ATMO 757858 and by the CNES. O.V. thanks the CNRS/INSU Programme National de Planétologie (PNP) and CNES for funding support.

Software: PASTIS (Díaz et al. 2014), Iraclis (Tsiaras et al. 2018), PyLightcurve (Tsiaras et al. 2016b), RATE (Cubillos et al. 2019), TauREx3 (Al-Refaie et al. 2019), Multinest (Feroz et al. 2009), ARIELRad (Mugnai et al. 2020), ExoWebb (B. Edwards et al. 2020, in preparation), emcee (Foreman-Mackey et al. 2013), Astropy (Astropy Collaboration et al. 2018), h5py (Collette 2013), Matplotlib (Hunter 2007), Numpy (Oliphant 2006), Imfit (Newville et al. 2019).

\section{Appendix Additional Figures}

In this appendix, in Figure A1 we show the results of the white-light-curve analysis for the transits not reported in Figure 1 for both the two exoplanets analyzed in this work, while in Figure A2 we plot the HD 3167c's orbits that showed contamination from HD 3167b. The last figure of the appendix (Figure A3) shows the posterior distribution we obtained by including also $\mathrm{N}_{2}$ in the retrieval analysis of HD 106315c. 

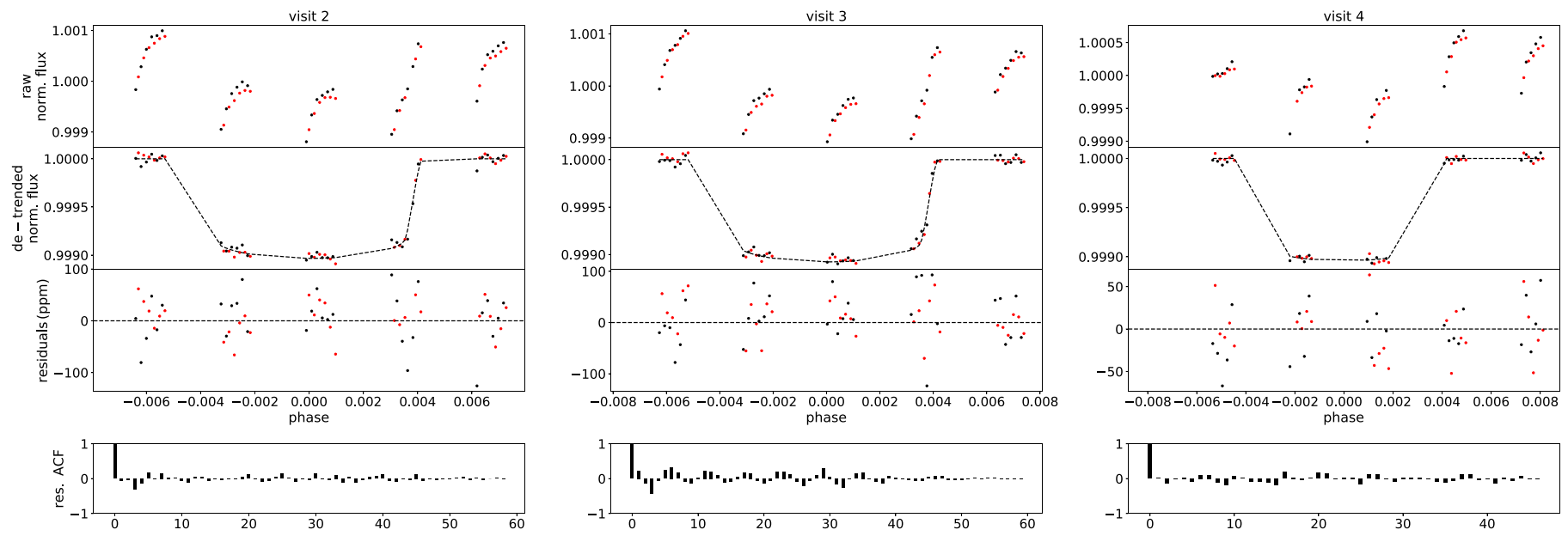

(a)
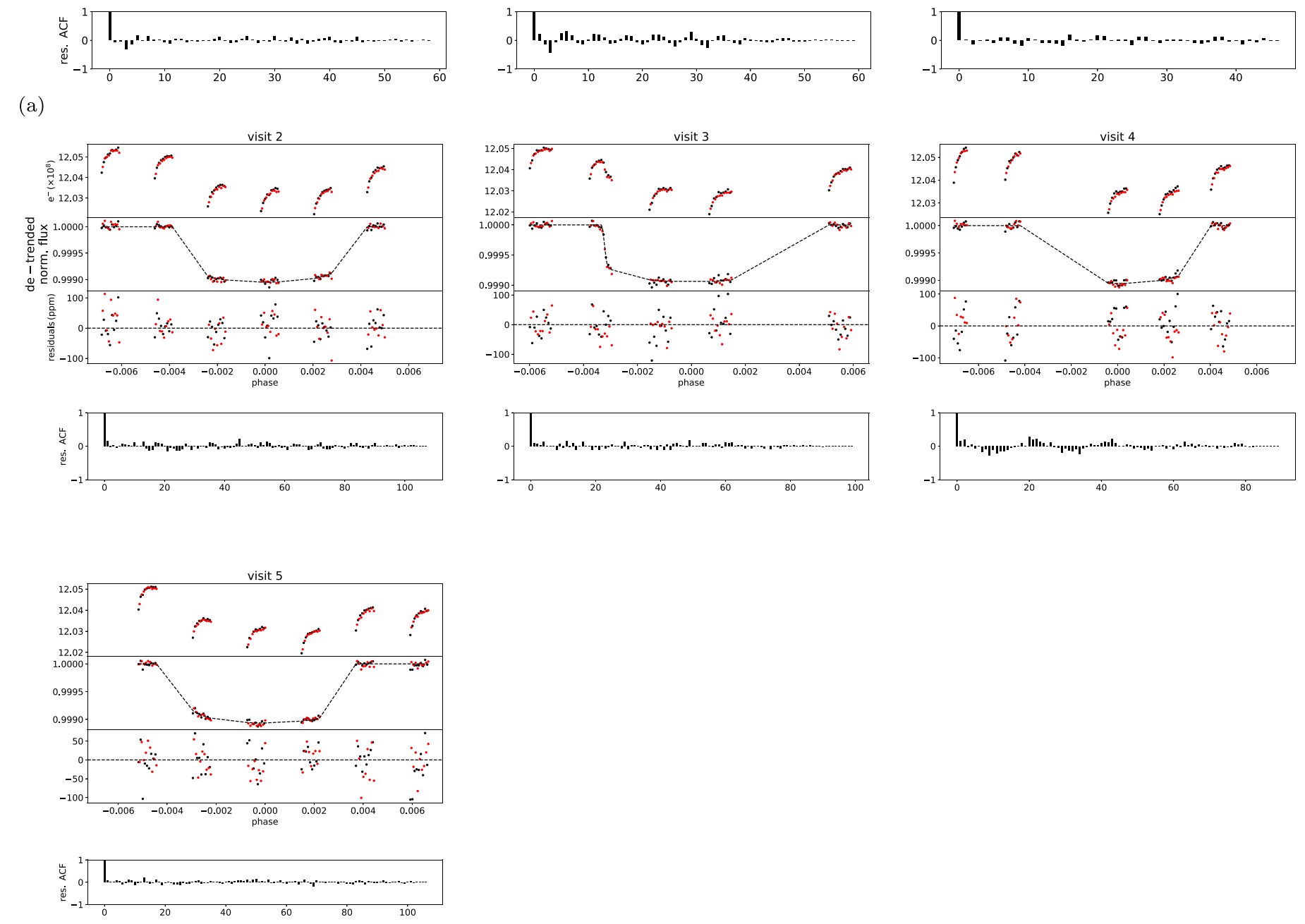

(b)

Figure A1. Same as Figure 1, but for the other transits for both (a) HD 106315c and (b) HD 3167c. 

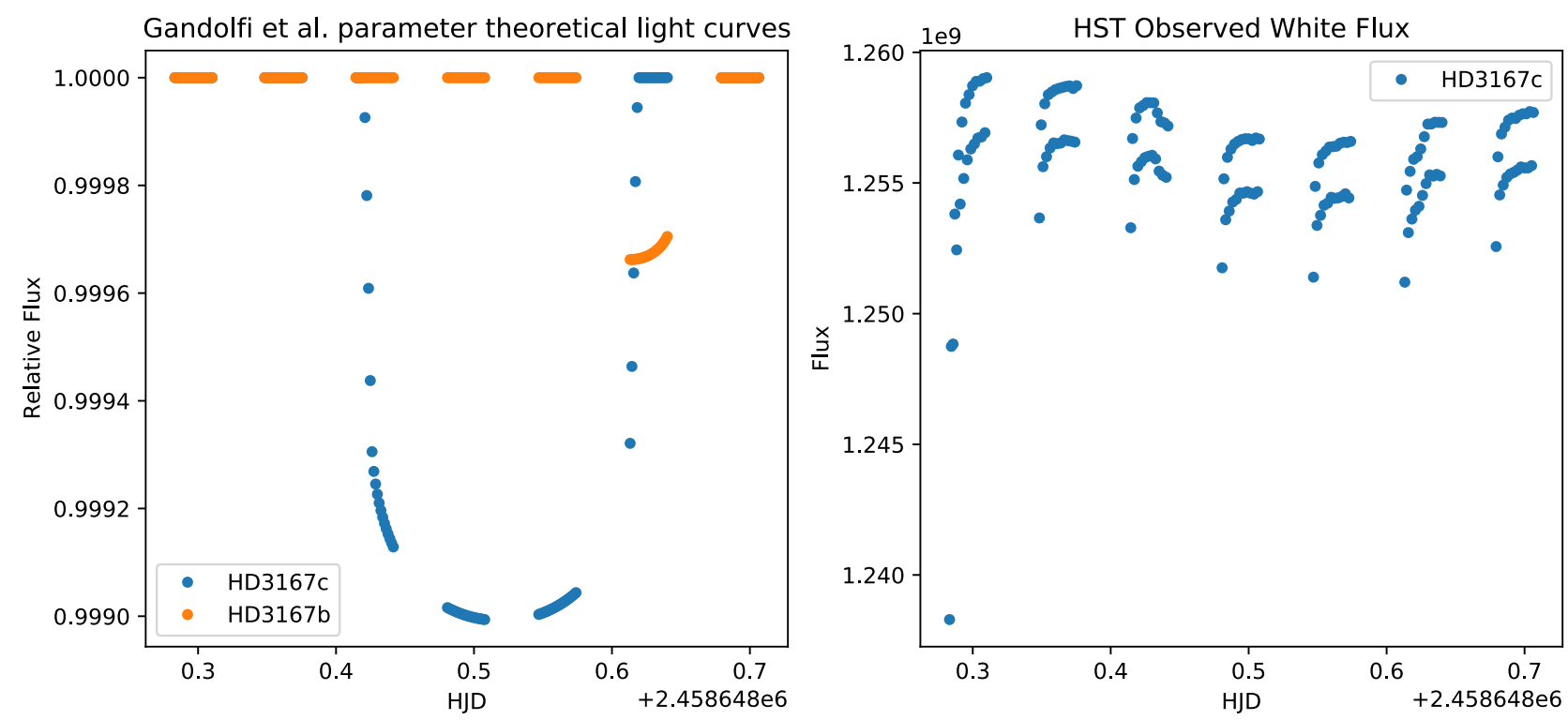

(a)
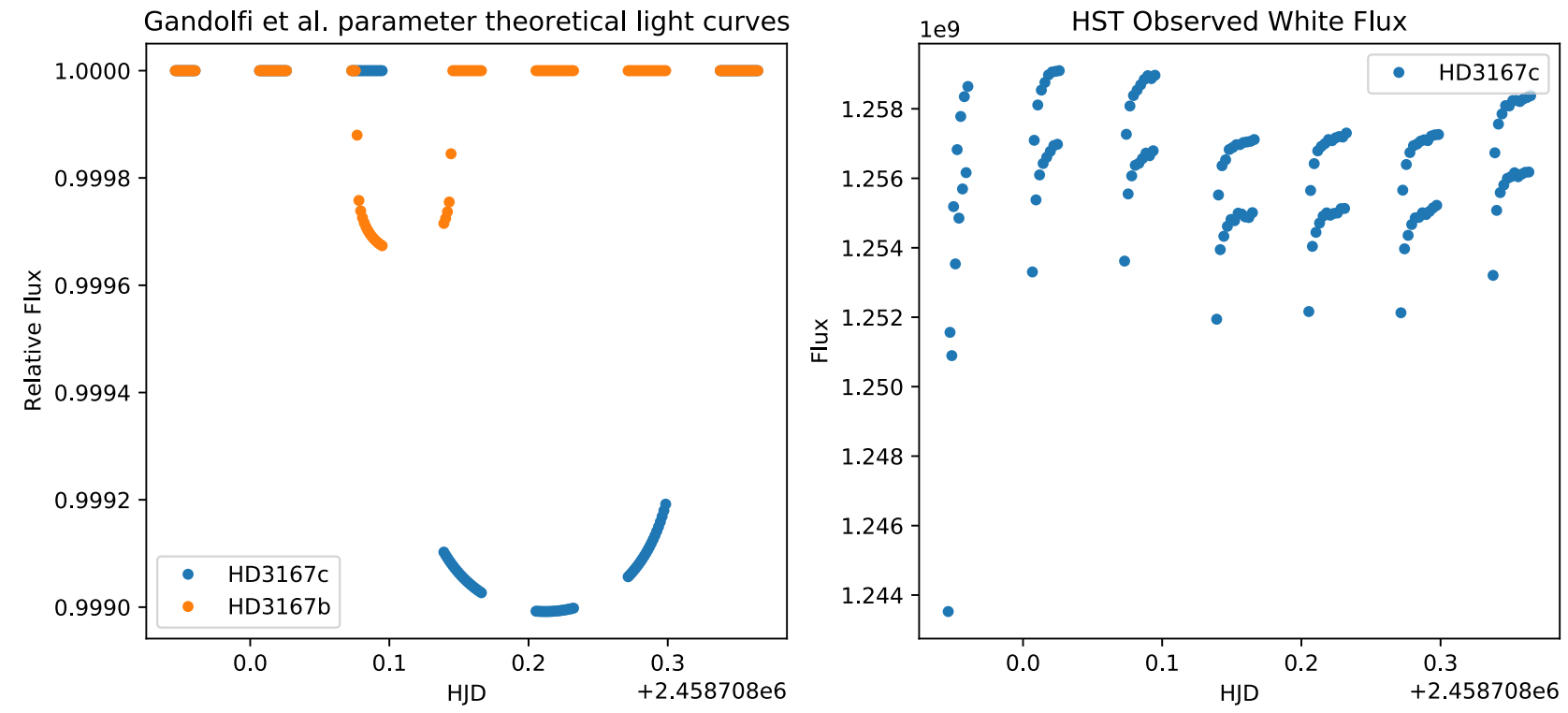

(b)

Figure A2. HD 3167c's orbits that showed contamination from HD 3167b. Visit 3 and the impact on its sixth orbit are shown in panel (a), while visit 4 and the contamination of its third orbit are shown in panel (b). 


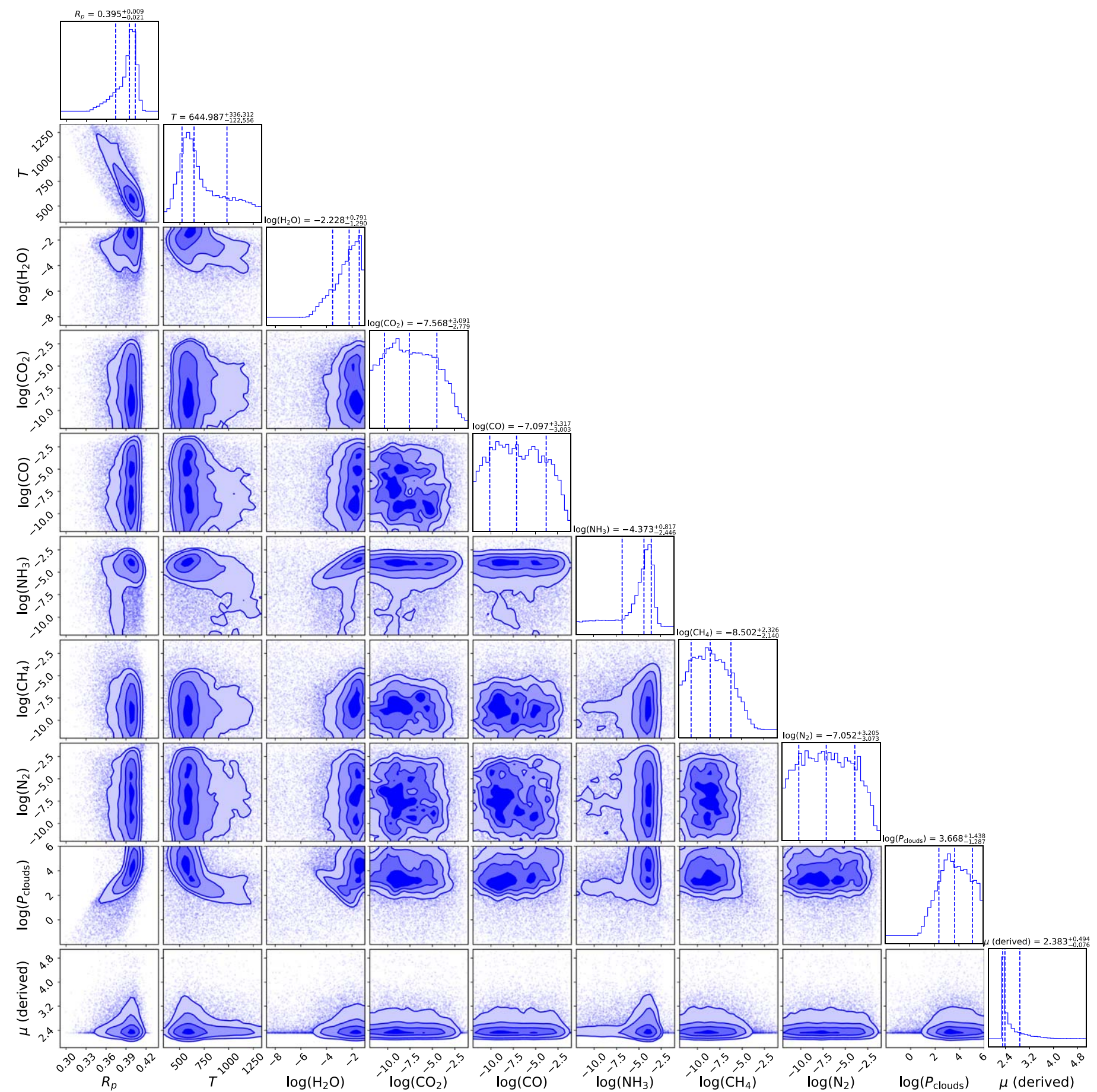

Figure A3. HD 106315c's posterior distribution including also $\mathrm{N}_{2}$ in the full chemical scenario. As this figure shows, the inclusion of nitrogen does not affect the mean molecular weight. Moreover, the detection of $\mathrm{NH}_{3}$ remains around $10^{-4}$. 


\section{ORCID iDs}

Gloria Guilluy @ https://orcid.org/0000-0002-1259-2678 Amélie Gressier (1) https://orcid.org/0000-0003-0854-3002 Alexandre Santerne (1) https://orcid.org/0000-0002-3586-1316 Billy Edwards (1) https://orcid.org/0000-0002-5494-3237 Quentin Changeat (10 https://orcid.org/0000-0001-6516-4493 Nour Skaf (1) https://orcid.org/0000-0002-9372-5056 Ahmed Al-Refaie (1) https://orcid.org/0000-0003-2241-5330 Michelle Fabienne Bieger (ib https://orcid.org/0000-00019166-3042

Mario Morvan (1) https://orcid.org/0000-0001-8587-2112 Lorenzo V. Mugnai (i) https://orcid.org/0000-0002-9007-9802 William Pluriel (10 https://orcid.org/0000-0001-7272-0363 Tiziano Zingales 나 https://orcid.org/0000-0001-6880-5356 Kai Hou Yip (1) https://orcid.org/0000-0002-9616-1524 Benjamin Charnay (i) https://orcid.org/0000-0003-0977-6545 Jérémy Leconte (i) https://orcid.org/0000-0002-3555-480X Pierre Drossart $(1)$ https://orcid.org/0000-0001-7261-0228 Alessandro Sozzetti (ib https://orcid.org/0000-0002-7504-365X Emmanuel Marcq (1) https://orcid.org/0000-0002-1924-641X Angelos Tsiaras (D https://orcid.org/0000-0003-3840-1793 Olivia Venot (1) https://orcid.org/0000-0003-2854-765X Jean-Philippe Beaulieu (1) https://orcid.org/0000-00030014-3354

\section{References}

Abel, M., Frommhold, L., Li, X., \& Hunt, K. L. 2011, JPCA, 115, 6805 Abel, M., Frommhold, L., Li, X., \& Hunt, K. L. 2012, JChPh, 136, 044319 Allart, R., Bourrier, V., Lovis, C., et al. 2018, Sci, 362, 1384

Al-Refaie, A. F., Changeat, Q., Waldmann, I. P., \& Tinetti, G. 2019, arXiv: 1912.07759

Astropy Collaboration, Price-Whelan, A. M., Sipőcz, B. M., et al. 2018, AJ, 156,18

Awiphan, S., Kerins, E., Pichadee, S., et al. 2016, MNRAS, 463, 2574 Barros, S. C. C., Gosselin, H., Lillo-Box, J., et al. 2017, A\&A, 608, A25 Batalha, N. M., Rowe, J. F., Bryson, S. T., et al. 2013, ApJS, 204, 24 Benneke, B., Knutson, H. A., Lothringer, J., et al. 2019a, NatAs, 3, 813 Benneke, B., \& Seager, S. 2013, ApJ, 778, 153

Benneke, B., Wong, I., Piaulet, C., et al. 2019b, ApJL, 887, L14

Borucki, W. J., Koch, D. G., Basri, G., et al. 2011, ApJ, 736, 19

Bourrier, V., Lecavelier des Etangs, A., Ehrenreich, D., et al. 2018, A\&A, 620, A147

Bourrier, V., Lecavelier des Etangs, A., Ehrenreich, D., Tanaka, Y. A., \& Vidotto, A. A. 2016, A\&A, 591, A121

Brandl, B. R., Absil, O., Agócs, T., et al. 2018, Proc. SPIE, 10702, 107021 U Brown, T. M., Charbonneau, D., Gilliland, R. L., Noyes, R. W., \& Burrows, A. 2001, ApJ, 552, 699

Caldas, A., Leconte, J., Selsis, F., et al. 2019, A\&A, 623, A161

Chachan, Y., Knutson, H. A., Gao, P., et al. 2019, AJ, 158, 244

Changeat, Q., Edwards, B., Waldmann, I. P., \& Tinetti, G. 2019, ApJ, 886, 39

Christiansen, J. L., Vanderburg, A., Burt, J., et al. 2017, AJ, 154, 122

Claret, A. 2000, A\&A, 363, 1081

Collette, A. 2013, Python and HDF5 (Sebastopol, CA: O'Reilly Media)

Cowan, N. B., Greene, T., Angerhausen, D., et al. 2015, PASP, 127, 311

Crossfield, I. J. M., Barman, T., \& Hansen, B. M. S. 2011, ApJ, 736, 132

Crossfield, I. J. M., Ciardi, D. R., Isaacson, H., et al. 2017, AJ, 153, 255

Crossfield, I. J. M., \& Kreidberg, L. 2017, AJ, 154, 261

Cubillos, P. E., Blecic, J., \& Dobbs-Dixon, I. 2019, ApJ, 872, 111

Díaz, R. F., Almenara, J. M., Santerne, A., et al. 2014, MNRAS, 441, 983

Dressing, C. D., \& Charbonneau, D. 2013, ApJ, 767, 95

Edwards, B., Changeat, Q., Baeyens, R., et al. 2020, AJ, 160, 8

Edwards, B., Mugnai, L., Tinetti, G., Pascale, E., \& Sarkar, S. 2019a, AJ, 157,242

Edwards, B., Rice, M., Zingales, T., et al. 2019b, ExA, 47, 29

Fanson, J., McCarthy, P. J., Bernstein, R., et al. 2018, Proc. SPIE, 10700, 1070012

Feroz, F., Hobson, M. P., \& Bridges, M. 2009, MNRAS, 398, 1601

Fisher, C., \& Heng, K. 2018, MNRAS, 481, 4698
Fletcher, L. N., Gustafsson, M., \& Orton, G. S. 2018, ApJS, 235, 24

Foreman-Mackey, D., Hogg, D. W., Lang, D., \& Goodman, J. 2013, PASP, 125,306

Fraine, J., Deming, D., Benneke, B., et al. 2014, Natur, 513, 526

Fressin, F., Torres, G., Charbonneau, D., et al. 2013, ApJ, 766, 81

Fulton, B., Petigura, E., Howard, A., et al. 2017, AJ, 154, 109

Fulton, B. J., \& Petigura, E. A. 2018, AJ, 156, 264

Gandolfi, D., Barragán, O., Hatzes, A. P., et al. 2017, AJ, 154, 123

Greene, T. P., Line, M. R., Montero, C., et al. 2016, ApJ, 817, 17

Harpsøe, K. B. W., Hardis, S., Hinse, T. C., et al. 2013, A\&A, 549, A10

Houk, N., \& Swift, C. 1999, Michigan Catalogue of Two-dimensional Spectral Types for the HD Stars, Vol. 5 (Ann Arbor, MI: Univ. Michigan)

Howard, A. W., Marcy, G. W., Bryson, S. T., et al. 2012, ApJS, 201, 15

Howarth, I. D. 2011, MNRAS, 413, 1515

Hunter, J. D. 2007, CSE, 9, 90

Kass, R. E., \& Raftery, A. E. 1995, Journal of the American Statistical Association, 90, 773

Knutson, H. A., Benneke, B. D., \& Homeier, D. 2014a, Natur, 505, 66

Knutson, H. A., Dragomir, D., Kreidberg, L., et al. 2014b, ApJ, 794, 155

Kreidberg, L., Bean, J. L., Désert, J. M., et al. 2014a, Natur, 505, 69

Kreidberg, L., Bean, J. L., Désert, J.-M., et al. 2014b, ApJL, 793, L27

Kreidberg, L., Mollière, P., Crossfield, I. J. M., et al. 2020, arXiv:2006.07444

Kundurthy, P., Agol, E., Becker, A. C., et al. 2011, ApJ, 731, 123

Kurucz, R. L. 1970, Atlas: A Computer Program for Calculating Model Stellar Atmospheres, SAO Special Report, 309

Léger, A., Selsis, F., Sotin, C., et al. 2004, Icar, 169, 499

Lendl, M., Ehrenreich, D., Turner, O. D., et al. 2017, A\&A, 603, L5

Li, G., Gordon, I. E., Rothman, L. S., et al. 2015, ApJS, 216, 15

Libby-Roberts, J. E., Berta-Thompson, Z. K., Désert, J. M., et al. 2020, AJ, 159,57

Lodders, K., \& Fegley, B. J. 2006, in Chemistry of Low Mass Substellar Objects, ed. J. W. Mason (Chichester: Praxis Publishing Ltd.), 1

MacDonald, R. J., Goyal, J. M., \& Lewis, N. K. 2020, ApJL, 893, L43

MacDonald, R. J., \& Madhusudhan, N. 2019, MNRAS, 486, 1292

Maciejewski, G., Niedzielski, A., Nowak, G., et al. 2014, AcA, 64, 323

Madhusudhan, N., \& Seager, S. 2011, ApJ, 729, 41

Mansfield, M., Bean, J. L., Oklopčić, A., et al. 2018, ApJL, 868, L34

Markwardt, C. B. 2009, in ASP Conf. Ser. 411, Astronomical Data Analysis Software and Systems XVIII, ed. D. A. Bohlender, D. Durand, \& P. Dowler (San Fransisco, CA: ASP), 251

Mikal-Evans, T., Crossfield, I. J. M., Benneke, B., et al. 2020, arXiv:2011. 03470

Morley, C. V., Fortney, J. J., Marley, M. S., et al. 2012, ApJ, 756, 172

Moses, J., Cavalié, T., Fletcher, L. N., \& Roman, M. T. 2020, arXiv:2006. 11367

Moses, J. I. 2014, RSPTA, 372, 20130073

Mousis, O., Deleuil, M., Aguichine, A., et al. 2020, ApJL, 896, L22

Mugnai, L. V., Pascale, E., Edwards, B., Papageorgiou, A., \& Sarkar, S. 2020, ExA, 50, 303

Newville, M., Otten, R., Nelson, A., et al. 2019, lmfit/lmfit-py 1.0.0, v1.0.0, Zenodo, doi: $10.5281 /$ zenodo. 3588521

Oliphant, T. E. 2006, A Guide to NumPy, Vol. 1 (Spanish Fork, UT: Trelgol Publishing)

Petigura, E. A., Howard, A. W., \& Marcy, G. W. 2013, PNAS, 110, 19273

Petigura, E. A., Howard, A. W., Marcy, G. W., et al. 2017, AJ, 154, 107

Pluriel, W., Whiteford, N., Edwards, B., et al. 2020a, AJ, 160, 112

Pluriel, W., Zingales, T., Leconte, J., \& Parmentier, V. 2020b, A\&A, 636, A66

Polyansky, O. L., Kyuberis, A. A., Zobov, N. F., et al. 2018, MNRAS, 480, 2597

Rocchetto, M., Waldmann, I. P., Venot, O., Lagage, P.-O., \& Tinetti, G. 2016, ApJ, 833, 120

Rodriguez, J. E., Zhou, G., Vanderburg, A., et al. 2017, AJ, 153, 256

Rogers, L. A. 2015, ApJ, 801, 41

Rogers, L. A., Bodenheimer, P., Lissauer, J. J., \& Seager, S. 2011, ApJ, 738, 59

Rogers, L. A., \& Seager, S. 2010a, ApJ, 712, 974

Rogers, L. A., \& Seager, S. 2010b, ApJ, 716, 1208

Rothman, L. S., Gordon, I. E., Barber, R. J., et al. 2010, JQSRT, 111, 2139

Sarkis, P., Henning, T., Kürster, M., et al. 2018, AJ, 155, 257

Skaf, N., Bieger, M. F., Edwards, B., et al. 2020, AJ, 160, 109

Skidmore, W., Anupama, G. C., \& Srianand, R. 2018, arXiv:1806.02481

Stassun, K. G., Collins, K. A., \& Gaudi, B. S. 2017, AJ, 153, 136

Tinetti, G., Drossart, P., Eccleston, P., et al. 2018, ExA, 46, 135

Tsiaras, A., Rocchetto, M., Waldmann, I. P., et al. 2016a, ApJ, 820, 99

Tsiaras, A., Waldmann, I., Rocchetto, M., et al. 2016b, pylightcurve: Exoplanet Lightcurve Model, Astrophysics Source Code Library, ascl:1612.018 
Tsiaras, A., Waldmann, I. P., Rocchetto, M., et al. 2016c, ApJ, 832, 202

Tsiaras, A., Waldmann, I. P., Tinetti, G., Tennyson, J., \& Yurchenko, S. N. 2019, NatAs, 3, 1086

Tsiaras, A., Waldmann, I. P., Zingales, T., et al. 2018, AJ, 155, 156

Valencia, D., O'Connell, R. J., \& Sasselov, D. 2006, Icar, 181, 545

van Grootel, V., Gillon, M., Valencia, D., et al. 2014, ApJ, 786, 2

Vanderburg, A., Bieryla, A., Duev, D. A., et al. 2016, ApJL, 829, L9

Venot, O., Cavalié, T., Bounaceur, R., et al. 2020, A\&A, 634, A78
Waldmann, I. P., Rocchetto, M., Tinetti, G., et al. 2015a, ApJ, 813, 13

Waldmann, I. P., Tinetti, G., Rocchetto, M., et al. 2015b, ApJ, 802, 107

Yurchenko, S. N., Barber, R. J., \& Tennyson, J. 2011, MNRAS, 413, 1828

Yurchenko, S. N., \& Tennyson, J. 2014, MNRAS, 440, 1649

Zeng, L., Jacobsen, S. B., Sasselov, D. D., et al. 2019, PNAS, 116, 9723

Zeng, L., \& Sasselov, D. 2013, PASP, 125, 227

Zeng, L., Sasselov, D. D., \& Jacobsen, S. B. 2016, ApJ, 819, 127

Zhou, G., Rodriguez, J. E., Vanderburg, A., et al. 2018, AJ, 156, 93 